\title{
Stability of Symmetric and Unsymmetric Thin Liquid Films to Short and Long Wavelength Perturbations
}

\author{
CHARLES MALDARELLI ${ }^{1}$ \\ Department of Chemical Engineering, Columbia University, New York, New York 10027 \\ RAKESH K. JAIN ${ }^{2}$ \\ Department of Chemical Engineering, Carnegie-Mellon University, Pittsburgh, Pennsylvania 15213 \\ IVAN B. IVANOV \\ Department of Physical Chemistry, University of Sofia, Bulgaria
}

AND

ELI RUCKENSTEIN

Faculty of Engineering and Applied Sciences, State University of New York, Buffalo, New York 14214

Received October 20, 1979; accepted March 8, 1980

\begin{abstract}
The stability of thin $(<100 \mathrm{~nm})$ symmetrical and unsymmetrical membranes, assimilated with viscous liquids, to short and long wavelength perturbations is investigated. The asymmetry is due to the two different viscous phases surrounding the membrane and to the different interfacial tensions on the two faces of the membrane. The cell membrane is a case for which the present treatment is of significance. The dynamics of the membrane to perturbations is described by the Navier-Stokes equation modified with a body force which accounts for the fact that the range of the interaction forces is larger than the thickness of the film. The body force is computed assuming pair-wise additivity and accounting for the deformation of the interface produced by the perturbation. General dispersion equations are derived, and these equations describe the squeezing and stretching modes of perturbations. The growth coefficient is expressed as a function of the wavelength for various ratios of the viscosities of the two surrounding phases and various values of the two interfacial tensions. In the limiting cases of interfacial tension ratio equal to unity and wavelength large compared to the thickness of the film results of the previous investigators are obtained. For the symmetrical case expressions are derived for the critical and dominant wavelength of the squeezing and stretching modes. An application of the results to a cell membrane shows that the growth of the instability is dominated in this case by the stretching mode since the time scale of growth of the perturbations is four orders of magnitude less than that in the squeezing mode. For unsymmetrical systems the effect of differences between the interfacial tensions on the two faces on the ratio of the amplitudes of perturbations on the two faces is investigated. The results show in what manner differences in interfacial tension convey amplified or damped messages across the membrane.
\end{abstract}

\section{INTRODUCTION}

The study of surface wave-induced hydrodynamic instabilities in thin $(O(10-$

\footnotetext{
${ }^{1}$ Present address: Department of Chemical Engineering, City College, New York, N. Y. 10031.

${ }^{2}$ Author to whom reprint requests should be addressed.
}

$100 \mathrm{~nm}$ )) liquid films has been the focus of a number of investigations by several workers (1-18). Although the motivations behind these investigations are diverse, clearly the most significant is the importance of this subject toward the understanding of particle coalescence phenomena in a continuous 


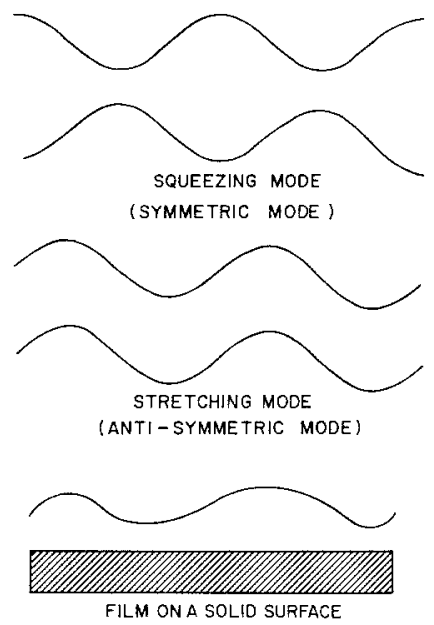

FIG. 1. Various modes of instability of a thin liquid film. In the squeezing mode, the corrugations at one interface of the film is completely out of phase with the corrugations at the other interface $(\phi=\pi)$. In the stretching mode this phase difference is zero $(\phi=0)$. When the film is supported on a solid surface, only one interface is corrugated.

liquid phase and the stability of the cell membrane.

In a dispersion, the approach of two particles (e.g., gas bubbles or liquid droplets) of the dispersed phase creates a radially bounded film which subsequently drains under the combined action of capillary suction at the plateau border and the disjoining pressure. If stabilizing surfactants are not present in sufficient amounts in the film system, the draining film will generally rupture at a thickness of the order of 10-100 $\mathrm{nm}$. According to deVries and later Scheludko (19), the rupture is caused by the undamped growth of mechanically or thermally induced corrugations in the film interfaces. These corrugations grow because the system becomes unstable to interfacial fluctuations at small film thicknesses.

The biological cell provides a contrasting example of a system in which a thin film plays a crucial role $(13,15,30)$. The cell membrane is a highly viscous lamella bounded by two different liquid phases (i.e., the intra- and extra-cellular fluids) and having different interfacial tensions on the two faces. Certain cell processes such as mobility, ingestion (via phagocytosis), and microvilli elaboration involve a deformation of the membrane. Reasons for the onset of these membrane deformation processes have not been fully elucidated, and it is hoped that a theoretical understanding of the stability of symmetric and unsymmetric, thin liquid films to short $(\lambda \leq h)$ and long $(\lambda>h)$ wavelength perturbations may provide insight into the origins of such processes.

The goal of this investigation is to examine the stability of pure, radially unbounded thin films bounded by two different liquids and having different interfacial tensions on the two faces. A short review of previous work is in order. Felderhof (2) analyzed the dynamics of a free film composed of an inviscid fluid and considered in detail both the squeezing and stretching normal modes (Fig. 1). This study was later extended by Sche (16) and Sche and Fijnaut (17) to include the effect of liquid viscosity. Ruckenstein and Jain (8) investigated the stability of the squeezing mode of a free film, but the major part of their investigations $(8,11)$ was concerned with a film on a solid substrate (Fig. 1). Lucassen et al. $(5,6)$ formally treated the stability of a liquid film bounded by two different viscous phases, but only derived an explicit dispersion equation for the squeezing mode of a symmetrical film system (i.e., a system in which the film is bounded by the same phase and in which gravity is neglected). Finally, Joosten et al. (14) extended the analysis of Lucassen et al. to a free film by considering the stretching mode of the symmetrical system. However, the dispersion equations obtained by Lucassen et al. and Joosten et al. are strictly applicable only to long wavelength interfacial disturbances (i.e., disturbances with Fourier component wavelengths which are much larger than the thickness of the film) because of the expansion method they employ to account for the 
influence of the long-range van der Waals interaction on the film dynamics (see below). In the work presented here, we will examine the stability characteristics of thin films for short as well as long wavelengths.

An important aspect of studies concerning the dynamics of thin films is the procedure utilized to account for the influence of the long-range van der Waals force on the film stability. The consideration of this force is essential: The maximum range of the van der Waals interaction $(O(100 \mathrm{~nm}))$ is of the same order of magnitude as the characteristic film thickness, and consequently energy inhomogeneities are created by this interaction in the film and in the adjoining phases. (This same phenomenon also occurs in the transition region between two bulk phases and is usually analyzed through the concept of surface tension.) Two different procedures have been utilized to account for the influence of the van der Waals interaction on the film dynamics: a disjoining pressure approach $(3,4,20,21)$ and a body force procedure $(2,8,11)$.

In the disjoining pressure approach, the film and the surrounding phases are treated as energetically homogeneous (with respect to the van der Waals interaction) up to the dividing surfaces which define the interfaces of the film. (This defines the idealized system.) The inhomogeneity caused by the van der Waals interaction is incorporated as a surface excess energy in a procedure analogous to the thermodynamic treatment of interfacial tension. Using this approach, one can show that the normal component of the stress tensor (of the idealized system) suffers a discontinuity (the disjoining pressure) at the dividing surface of the film.

The disjoining pressure approach has the advantage that all types of intermolecular forces (i.e., dispersion, Keesom, and Debye-Falkenhagen forces) which contribute to the long-range van der Waals interaction can easily be formulated in the expression for the disjoining pressure by utilizing Lifschitz's theory. However, as shown below for the stability analysis, an expression for the disjoining pressure of an interfacially deformed film is required. In all previous works $(4-7,9,10,12,14)$, such an expression has been obtained by expanding the disjoining pressure of a plane parallel film in a power series with respect to the film thickness. This procedure is valid for long wavelength interfacial disturbances, but it is not valid for short wavelength disturbances.

In the body force approach, the influence of the van der Waals force is incorporated as a body force in the Navier-Stokes equation of motion. The force is derived from a potential which describes the energy of interaction (due to the van der Waals force) of molecules in an infinitesimal volume of continua with respect to the entire ensemble of molecules in the system. In the usual application of the body force approach, this potential is calculated via a microscopic Hamaker procedure in which the intermolecular potential is integrated over the system volume. Although an adequate approximation for describing the dispersion contribution to the van der Waals interaction, the Hamaker procedure is insufficient for incorporating the dipolar contributions (i.e., the Keesom and DebyeFalkenhagen forces) since these are incompatible with pair additivity. However, this need not be considered a serious limitation since ad hoc methods for incorporating the dipolar contributions into the Hamaker procedure have been developed (22).

Utilization of the Hamaker procedure to obtain the body force potential has two distinct advantages: First, in contrast to the disjoining pressure approach it can easily be formulated for any interfacially corrugated state of the film's interfaces and is therefore applicable to both short and long wavelength disturbance regimes. Second, it is computationally easier to formulate the Hamaker potential for an unsymmetrical film system (i.e., a film bounded by two 


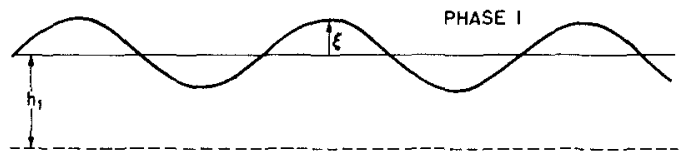

A

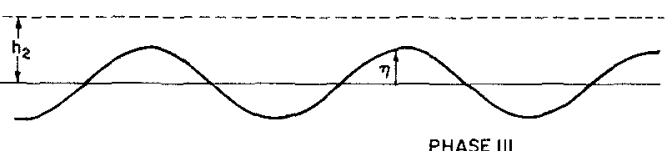

FIG. 2. The infinite system. The two interfaces, arbitrarily deformed, are separated by an infinite reservoir of phase II (region "A").

different viscous phases) than it is to calculate the disjoining pressure of such a system.

Since this investigation is concerned with both symmetrical and unsymmetrical film systems, the body force method, in conjunction with the Hamaker procedure to calculate the intermolecular potential, is utilized. Although in all studies in which the body force method has been used $(2,8,11$, $16,17)$ the Hamaker procedure has been employed to calculate the potential, no care has yet been taken regarding a systematic formulation of this potential for corrugated interfaces. Consequently this analysis begins with a systematic formulation of the Hamaker potential for an unsymmetrical, arbitrarily deformed film system. The basic equations for the first-order approximations for the field variables follow. These equations differ from those established previously through the expression of the body force which contains the effect of the corrugation of the interface and through different viscosities and interfacial tensions. A general dispersion equation is established in the next section which is valid for both short and long wavelength disturbances. The final sections of the paper examine the dispersion equation for symmetrical and unsymmetrical systems. First, the asymptotic forms of the symmetrical dispersion equations are derived for the stretching and squeezing modes in the long wavelength limit. Then, the symmetrical dispersion equations are analyzed numerically to examine the stability characteristics of the film to short wavelength perturbations. Finally, the dispersion equation for the unsymmetrical system is analyzed numerically to study the role of interfacial tension asymmetry on the film behavior. Wherever possible, results are used to explain available experimental data.

\section{FORMULATION OF THE BODY FORCE VIA A HAMAKER PROCEDURE}

To compute the long-range van der Waals interaction potential, it is useful to consider the state in which the interfaces of the film are arbitrarily deformed and located an infinite distance apart from each other (see Fig. 2). In this "infinite"' system, the interfaces are isolated and can be separately treated in the manner of Gibbs as dividing surfaces of zero thickness separating phases which are homogeneous in all intensive properties (e.g., density and energy) up to the dividing surfaces. The spatial inhomogeneities in energy (which are especially pronounced in the interfacial transition regions) caused by the van der Waals force are accounted for by treating the dividing surfaces as membranes in isotropic tension; by utilizing this procedure, the idealized Gibbs system is made mechanically equivalent to the real system and no body force is considered. (In what follows, the liquid comprising the film is referred to as phase II and the fluids comprising the upper and

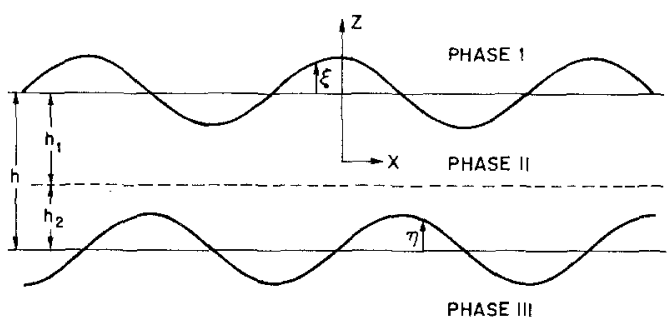

FIG. 3. The film system. Region " $A$ " has been removed to an infinite reservoir of phase II. The origin of the coordinate system is on the midplane of the unperturbed film. 
lower semi-infinite media are denoted by phases I and III, respectively. The interfaces between phases I and II and II and III are referred to as the upper (u) and lower (l) interfaces, respectively.)

When the interfaces are brought close together by removing (to an infinite reservoir of phase II) region "A" of Fig. 2 to form the film system (Fig. 3), the molecules in the film and the adjoining phases experience a change in potential energy. The change in potential energy is derived by first computing the potential energy of interaction (due to the van der Waals force) of molecules in an infinitesimal volume of continua with respect to (i) the rest of the molecules in the film system (Fig. 3) and (ii) the rest of the molecules in the infinite system (Fig. 2) and then subtracting the energy obtained in (ii) from that calculated in (i). This difference is termed the excess van der Waals potential; the negative gradient of this potential is the body force which is introduced into the equation of motion.

In the Hamaker procedure, the potential energy of interaction is computed by integrating the intermolecular potential $u(r)$ (the mutual potential energy of two molecules separated by a distance $r$ ) over the system volume. Thus in this procedure, the excess van der Waals potential admits the following integral representation:

$$
\begin{aligned}
W(\mathbf{r}, t) & =\int_{\substack{\text { film } \\
\text { system }}} w\left(\left|\mathbf{r}-\mathbf{r}^{\prime}\right|^{1 / 2}\right) \rho\left(\mathbf{r}^{\prime}, t\right) d \mathbf{r}^{\prime} \\
& -\int_{\substack{\text { infinite } \\
\text { system }}} w\left(\left|\mathbf{r}-\mathbf{r}^{\prime}\right|^{1 / 2}\right) \rho\left(\mathbf{r}^{\prime}, t\right) d \mathbf{r}^{\prime} .
\end{aligned}
$$

In Eq. [1] $t$ represents time, $\rho\left(\mathbf{r}^{\prime}, t\right)$ is the mass density at the source point $\mathbf{r}^{\prime}, W(\mathbf{r}, t)$ is the excess van der Waals potential (per unit mass) at the field point $\mathbf{r}$, and $w(r)$ is the intermolecular potential $u(r)$ divided by the masses (per molecule) of the molecules located at $\mathbf{r}$ and $\mathbf{r}^{\prime}$.
To formulate an explicit expression for the excess potential in terms of the Cartesian coordinate system diagrammed in Fig. 3, the following functions prove essential:

$$
\begin{gathered}
F_{i, j}(s)=\rho^{i} \rho^{j} \int_{s}^{\infty} 2 \pi r w_{i, j}(r) d r \\
H_{i, j}^{1}(x, y, v, t)=\rho^{i} \rho^{j} \int_{-\infty}^{\infty} \int_{-\infty}^{\infty} \int_{0}^{\xi(\alpha, \beta, t)} \\
w_{i, j}\left(\left((v-\gamma)^{2}+(x-\alpha)^{2}\right.\right. \\
\left.\left.+(y-\beta)^{2}\right)^{1 / 2}\right) d \gamma d \beta d \alpha \\
H_{i, j}^{2}(x, y, v, t)=\rho^{i} \rho^{j} \int_{-\infty}^{\infty} \int_{-\infty}^{\infty} \int_{0}^{\eta(\alpha, \beta, t)} \\
w_{i, j}\left(\left((v-\gamma)^{2}+(x-\alpha)^{2}\right.\right. \\
\left.\left.+(y-\beta)^{2}\right)^{1 / 2}\right) d \gamma d \beta d \alpha .
\end{gathered}
$$

The variables $\xi$ and $\eta$ define the interfacial perturbations (see Fig. 3). The function $F_{i, j}(s)$ represents the potential energy (per unit volume) at a point $\mathbf{r}$ in phase $i$ due to an infinite plane of phase $j$ that is located a perpendicular distance $s$ from $\mathbf{r}$. The function $H_{i, j}^{1}(x, y, v, t)\left(H_{i, j}^{2}(x, y, v, t)\right)$ is the perturbation in the potential energy (per unit volume) at a point in phase $i$, with coordinates $x, y$ and located a distance $|v|$ from the unperturbed upper (lower) interface, caused by the displacement of phase $j$ with the corrugation of the interface. In Eqs. [2]- [4] density variations have been neglected since the fluids comprising the film system are assumed incompressible.

With the aid of these functions, the integral representation of the excess potential can be written with reference to the $x, y, z$ Cartesian coordinate system of Fig. 3.

$$
\begin{aligned}
\rho^{\mathrm{I}} W^{\mathrm{I}}(x, y, z, t) & =\int_{z+h / 2}^{\infty}\left(F_{\mathrm{I}, \mathrm{III}}(u)-F_{\mathrm{I}, \mathrm{II}}(u)\right) d u \\
& +H_{\mathrm{I}, \mathrm{II}}^{2}\left(x, y, z+\frac{h}{2}, t\right) \\
& -H_{\mathrm{I}, \mathrm{II}}^{2}\left(x, y, z+\frac{h}{2}, t\right)
\end{aligned}
$$




$$
\begin{aligned}
& \rho^{\mathrm{II}} W^{\mathrm{II}}(x, y, z, t) \\
& =\int_{z+h / 2}^{\infty}\left(F_{\mathrm{II}, \mathrm{III}}(u)-F_{\mathrm{II}, \mathrm{II}}(u)\right) d u \\
& +H_{\mathrm{II}, \mathrm{III}}^{2}\left(x, y, z+\frac{h}{2}, t\right) \\
& -H_{\mathrm{II}, \mathrm{II}}^{2}\left(x, y, z+\frac{h}{2}, t\right) \\
& {\left[\xi(x, t)+\frac{h}{2}>z>\frac{h}{2}-h_{1}\right]} \\
& \rho^{\mathrm{II}} W^{\mathrm{II}}(x, y, z, t)=\int_{h / 2-z}^{\infty}\left(F_{\mathrm{I}, \mathrm{II}}(u)\right. \\
& \left.-F_{\mathrm{II}, \mathrm{II}}(u)\right) d u+H_{\mathrm{II}, \mathrm{II}}^{1}\left(x, y, z-\frac{h}{2}, t\right) \\
& -H_{\mathrm{I}, \mathrm{II}}^{1}\left(x, y, z-\frac{h}{2}, t\right) \\
& {\left[\frac{h}{2}-h_{1}>z>\eta(x, t)-\frac{h}{2}\right]} \\
& \rho^{\mathrm{III}} W^{\mathrm{III}}(x, y, z, t)=\int_{h / 2-z}^{\infty}\left(F_{\mathrm{I}, \mathrm{III}}(u)\right. \\
& \left.-F_{\mathrm{II}, \mathrm{III}}(u)\right) d u+H_{\mathrm{II}, \mathrm{III}}^{1}\left(x, y, z-\frac{h}{2}, t\right) \\
& -H_{1, \mathrm{II}}^{1}\left(x, y, z-\frac{h}{2}, t\right) \text {. }
\end{aligned}
$$

In the above equations $h$ is the film thickness; $h_{1}$ is an arbitrary parameter utilized in dividing the film volume (see Figs. 2 and 3 ). The roman numeraled superscripts indicate the phase in which the scripted quantity is evaluated.

It is important to note that $W^{i}(x, y, z, t)$ is the excess van der Waals potential; the total potential is the sum of the excess potential and the reference potential. Thus

$$
\begin{gathered}
\rho^{\mathrm{I}} \bar{W}^{\mathrm{I}}=\rho^{\mathrm{I}} W^{\mathrm{I}}+\rho^{\mathrm{I}} W_{\infty(2)}^{\mathrm{I}} \\
\rho^{\mathrm{II}} \bar{W}^{\mathrm{II}}=\rho^{\mathrm{II}} W^{\mathrm{II}}+\rho^{\mathrm{II}} W_{\infty \propto(2)}^{\mathrm{II}} \\
\left(\xi(x, t)+\frac{h}{2}>z>\frac{h}{2}-h_{1}\right)
\end{gathered}
$$

$$
\begin{gathered}
\rho^{\mathrm{II}} \bar{W}^{\mathrm{II}}=\rho^{\mathrm{II}} W^{\mathrm{II}}+\rho^{\mathrm{II}} W_{\infty(1)}^{\mathrm{II}} \\
\left(\frac{h}{2}-h_{1}>z>\eta(x, t)-\frac{h}{2}\right) \\
\rho^{\mathrm{III}} \bar{W}^{\mathrm{III}}=\rho^{\mathrm{II}} W^{\mathrm{III}}+\rho^{\mathrm{III}} W_{\infty(1)}^{\mathrm{III}} .
\end{gathered}
$$

Here, $\bar{W}^{i}$ indicates the total potential; $W_{\infty(1)}^{i}$ and $W_{\infty(2)}^{i}$ are the reference potentials (the subscript 1 indicates that the I, II interface is infinitely far away and the subscript 2 indicates that the II, III interface is at infinity). Note that since the reference potential for $z>h / 2-h_{1}$ is taken different than that for $z<h / 2-h_{1}$, the excess potential is discontinuous at $z=h / 2-h_{1}$ even though the total potential in the film, $\bar{W}^{\text {II }}$, is continuous. However, the dispersion equation involves only the gradient of these potentials; the choice of the reference potentials does not create any problem, as will be evident in the next two sections.

\section{STABILITY ANALYSIS}

This investigation is concerned with the interfacial stability of an unbounded, nonthinning liquid film surrounded by two different viscous phases and having different interfacial tensions on the two faces. Since the film is not thinning, the base state is taken to be one in which the flow velocity is zero and the film interfaces are plane parallel. Infinitesimal perturbations are applied to the interfaces of the film, and the object of the analysis is to determine the stability of the resulting fluid motion. For simplicity, only two-dimensional motion is considered; the extension to three dimensions is discussed after Eq. [77].

The field equations which are necessary to describe the fluid motion are the continuity and the Navier-Stokes equations for an incompressible fluid $(23,24)$. The boundary conditions at the displaced interfaces are the continuity of velocity requirement, the kinematic relation, and the tangential and normal stress balances (24). Furthermore, the disturbance must decay 
to zero at an infinite distance from the perturbed interfaces.

In order to ascertain the stability of the fluid motion induced by the interfacial perturbations, the field equations and boundary conditions for the flow are solved by using perturbation methods (23). The field variables of the system are expanded in a series about their values in the base state. Since the initial disturbances are infinitesimal, it suffices to consider only linear terms. Therefore

$$
\begin{aligned}
& p^{j}=p_{0}^{j}(z)+\epsilon p_{1}^{j}(x, z, t) \\
& u^{j}=\epsilon u_{1}^{j}(x, z, t) \\
& v^{j}=\epsilon v_{1}^{j}(x, z, t)
\end{aligned}
$$

$$
\begin{aligned}
\xi & =\epsilon \xi_{1}(x, t) \\
\eta & =\epsilon \eta_{1}(x, t) \\
W^{j} & =W_{0}^{j}(z)+\epsilon W_{1}^{j}(x, z, t) .
\end{aligned}
$$

In the above set of equations, $\epsilon$ is a small, dimensionless parameter, and the subscripts " 0 " and " 1 " indicate the base state and first-order values, respectively, of the scripted quantities. The variables $p^{j}, u^{j}$, and $v^{j}$ denote, respectively, the pressure and the $x$ and $z$ components of the velocity vector (all of phase $j$ ).

The base state distribution for the excess van der Waals potential is obtained from Eqs. [5]- [8] by setting the $H_{i, j}^{1}$ and $H_{i, j}^{2}$ functions equal to zero. Thus

$$
\begin{aligned}
& \rho^{\mathrm{I}} W_{0}^{\mathrm{I}}(z)=\int_{z+h / 2}^{\infty}\left(F_{\mathrm{I}, \mathrm{III}}(u)-F_{\mathrm{I}, \mathrm{II}}(u)\right) d u \\
& \rho^{\mathrm{II}} W_{0}^{\mathrm{II}}(z)= \begin{cases}\int_{z+h / 2}^{\infty}\left(F_{\mathrm{II}, \mathrm{III}}(u)-F_{\mathrm{II}, \mathrm{II}}(u)\right) d u & \left(\frac{h}{2}>z>\frac{h}{2}-h_{1}\right) \\
\int_{h / 2-z}^{\infty}\left(F_{\mathrm{I}, \mathrm{II}}(u)-F_{\mathrm{II}, \mathrm{II}}(u)\right) d u & \left(\frac{h}{2}-h_{1}>z>-\frac{h}{2}\right)\end{cases} \\
& \rho^{\mathrm{III}} W_{0}^{\mathrm{III}}(z)=\int_{h / 2-z}^{\infty}\left(F_{\mathrm{I}, \mathrm{III}}(u)-F_{\mathrm{II}, \mathrm{III}}(u)\right) d u .
\end{aligned}
$$

Explicit expressions for the first-order excess van der Waals potential, $W_{1}^{j}$, are obtained by expanding the $H_{i, j}^{1}$ and $H_{i, j}^{2}$ functions in a power series about $\xi$ and $\eta$ equal to zero, and then substituting the first-order approximations for $\xi$ and $\eta$ (Eqs. [12] and [13]) into these expansions. The results for one-dimensional interfacial disturbance (two-dimensional flow) are:

$$
\begin{aligned}
& \rho^{\mathrm{I}} W_{1}^{\mathrm{I}}(x, z, t)=Q_{\mathrm{I}, \mathrm{II}}^{2}\left(x, z+\frac{h}{2}, t\right)-Q_{\mathrm{I}, \mathrm{II}}^{2}\left(x, z+\frac{h}{2}, t\right) \\
& \rho^{\mathrm{II}} W_{1}^{\mathrm{II}}(x, z, t)=\left\{\begin{array}{l}
Q_{\mathrm{II}, \mathrm{III}}^{2}\left(x, z+\frac{h}{2}, t\right)-Q_{\mathrm{II}, \mathrm{II}}^{2}\left(x, z+\frac{h}{2}, t\right)\left(\frac{h}{2}>z>\frac{h}{2}-h_{1}\right) \\
Q_{\mathrm{I} 1, \mathrm{II}}^{1}\left(x, z-\frac{h}{2}, t\right)-Q_{\mathrm{I}, \mathrm{II}}^{1}\left(x, z-\frac{h}{2}, t\right)\left(\frac{h}{2}-h_{1}>z>-\frac{h}{2}\right)
\end{array}\right. \\
& \rho^{\mathrm{III}} W_{1}^{\mathrm{III}}(x, z, t)=Q_{\mathrm{II}, \mathrm{II}}^{1}\left(x, z-\frac{h}{2}, t\right)-Q_{\mathrm{I}, \mathrm{II}}^{\mathrm{I}}\left(x, z-\frac{h}{2}, t\right)
\end{aligned}
$$


where

$$
\begin{array}{r}
Q_{i, j}^{1}(x, v, t)=\rho^{i} \rho^{j} \int_{-\infty}^{\infty} \int_{-\infty}^{\infty} \xi_{1}(\alpha, t) w_{i, j} \\
\left(\left(v^{2}+\xi^{2}+(x-\alpha)^{2}\right)^{1 / 2}\right) d \xi d \alpha \\
Q_{i, j}^{2}(x, v, t)=\rho^{i} \rho^{j} \int_{-\infty}^{\infty} \int_{-\infty}^{\infty} \eta_{1}(\alpha, t) w_{i, j} \\
\left(\left(v^{2}+\xi^{2}+(x-\alpha)^{2}\right)^{1 / 2}\right) d \xi d \alpha .
\end{array}
$$

\section{The Base State}

In the unperturbed (base) state the interfaces of the film are plane parallel and the flow velocity is equal to zero. Consequently the continuity equation and the entire set of boundary conditions (with the exception of the normal stress balances) reduce to identities, and the Navier-Stokes equation after integration, becomes:

$$
\begin{gathered}
p_{0}^{j}(z)+\rho^{j} g z+\rho^{j} W_{0}^{j}(z)=c^{j} \\
(j=\mathrm{I}, \mathrm{II}, \mathrm{III}) .
\end{gathered}
$$

In Eq. [23], $g$ is the acceleration of gravity; it is assumed that gravity acts in the negative $z$ direction (see Fig. 3). The only nontrivial boundary conditions, the normal stress balances, reduce to the following form:

$$
\begin{gathered}
p_{0}^{\mathrm{I}}\left(\frac{h}{2}\right)=p_{0}^{\mathrm{II}}\left(\frac{h}{2}\right), \\
p_{0}^{\mathrm{II}}\left(-\frac{h}{2}\right)=p_{0}^{\mathrm{III}}\left(-\frac{h}{2}\right) .
\end{gathered}
$$

In the base state, the total force density (excluding gravity) is equal to the sum of the pressure and the excess van der Waals potential; thus

$$
P_{0}^{j}(z)=p_{0}^{j}(z)+\rho^{j} W_{0}^{j}(z)
$$

where $P_{0}^{j}(z)$ denotes the gravity-excluded total force density of phase $j$. Owing to the continuity in the pressure at the two interfaces (Eqs. [24] and [25]), this force density suffers a discontinuity at $z= \pm h / 2$. Explicitly,

$$
\begin{aligned}
& P_{0}^{\mathrm{I}}\left(\frac{h}{2}\right)-P_{0}^{\mathrm{II}}\left(\frac{h}{2}\right)=\rho^{\mathrm{I}} W_{0}^{\mathrm{I}}\left(\frac{h}{2}\right) \\
&-\rho^{\mathrm{II}} W_{0}^{\mathrm{II}}\left(\frac{h}{2}\right)=\int_{h}^{\infty} F(u) d u \\
& P_{0}^{\mathrm{III}}\left(-\frac{h}{2}\right)-P_{0}^{\mathrm{II}}\left(-\frac{h}{2}\right)=\rho^{\mathrm{III}} W_{0}^{\mathrm{II}}\left(-\frac{h}{2}\right) \\
&-\rho^{\mathrm{II}} W_{0}^{\mathrm{II}}\left(-\frac{h}{2}\right)=\int_{h}^{\infty} F(u) d u
\end{aligned}
$$

where

$$
\begin{aligned}
F(u)=F_{\mathrm{I}, \mathrm{III}}(u) & +F_{\mathrm{II}, \mathrm{II}}(u) \\
& -F_{\mathrm{I}, \mathrm{II}}(u)-F_{\mathrm{II}, \mathrm{III}}(u) .
\end{aligned}
$$

The second equality in Eqs. [27] and [28] follows from Eqs. [15]-[17]. The function $F(u)$ can be expressed in the following integral form:

$$
F(u)=\int_{u}^{\infty} 2 \pi v \tilde{w}(v) d v
$$

where

$$
\begin{aligned}
\tilde{w}(r)= & \rho^{\mathrm{I}} \rho^{\mathrm{III}} w_{\mathrm{I}, \mathrm{III}}(r)+\rho^{\mathrm{II}} \rho^{\mathrm{II}} w_{\mathrm{II}, \mathrm{II}}(r) \\
& -\rho^{\mathrm{I}} \rho^{\mathrm{II}} w_{\mathrm{I}, \mathrm{II}}(r)-\rho^{\mathrm{II}} \rho^{\mathrm{III}} w_{\mathrm{II}, \mathrm{III}}(r) .
\end{aligned}
$$

Equations [27] and [28] indicate that the discontinuities in the force density at the two interfaces of the film are equal. This conclusion is valid despite the fact that the adjoining phases have not been assumed to be identical. Hence, the equality of Eqs. [27] and [28] suggests that these localized differences in the force density can be regarded as a characteristic of the entire film system. This difference is referred to here as the disjoining pressure $(\Pi(h))$ of a plane parallel infinite film. Thus:

$$
\Pi(h)=\int_{h}^{\infty} F(u) d u .
$$

\section{Explicit Solution for the First-Order Field Variables via Normal Mode Analysis}

The first-order approximations for the field variables are substituted into the field and boundary equations, and the resulting 
relations are simplified to first order in $\epsilon$. After performing these operations, the field equations and conditions at infinity become:

$$
\begin{gathered}
\frac{\partial u_{1}^{j}}{\partial x}+\frac{\partial v_{1}^{j}}{\partial z}=0 \\
\rho^{j} \frac{\partial u_{1}^{j}}{\partial t}=-\frac{\partial p_{1}^{j}}{\partial x}+\mu^{j} \nabla^{2} u_{1}^{j}-\rho^{j} \frac{\partial W_{1}^{j}}{\partial x} \\
\rho^{j} \frac{\partial v_{1}^{j}}{\partial t}=-\frac{\partial p_{1}^{j}}{\partial z}+\mu^{j} \nabla^{2} v_{1}^{j}-\rho^{j} \frac{\partial W_{1}^{j}}{\partial z} \\
(j=\mathrm{I}, \mathrm{II}, \mathrm{III}) \\
\lim _{z \rightarrow \infty} u_{1}^{\mathrm{I}}=\lim _{z \rightarrow \infty} v_{1}^{\mathrm{I}}=0
\end{gathered}
$$

$$
\lim _{z \rightarrow-\infty} u_{1}^{\mathrm{III}}=\lim _{z \rightarrow-\infty} v_{1}^{\mathrm{III}}=0 .
$$

The boundary conditions at the displaced interfaces are composed about the mathematical surfaces $z=\xi(x, t)+h / 2$ and $z=\eta(x, t)-h / 2$. The value of a field variable on either one of these surfaces can be obtained by expanding this variable in a Taylor series (in powers of $\xi$ or $\eta$ ) about its value on the mean plane of the interfacial displacements (i.e., $z= \pm h / 2$ ). This series expansion can then be expressed in ascending powers of $\epsilon$ by introducing the first order approximations for $\xi, \eta$ and the system variable into the series. Using this procedure, the first-order boundary conditions at the displaced surfaces become:

$$
\begin{aligned}
& v_{1}^{\mathrm{I}}=v_{1}^{\mathrm{II}} \\
& u_{1}^{\mathrm{I}}=u_{1}^{\mathrm{II}} \\
& v_{1}^{\mathrm{II}}=\frac{\partial \xi_{1}}{\partial t} \\
& \mu^{\mathrm{II}}\left(\frac{\partial u_{1}^{\mathrm{II}}}{\partial z}+\frac{\partial v_{1}^{\mathrm{II}}}{\partial x}\right)-\mu^{\mathrm{I}}\left(\frac{\partial u_{1}^{\mathrm{I}}}{\partial z}+\frac{\partial v_{1}^{\mathrm{I}}}{\partial x}\right)=0 \\
& -p_{1}^{\mathrm{II}}+2 \mu^{\mathrm{II}} \frac{\partial v_{1}^{\mathrm{II}}}{\partial z}+p_{1}^{\mathrm{I}}-2 \mu^{\mathrm{I}} \frac{\partial v_{1}^{\mathrm{I}}}{\partial z}+\xi_{1}\left(\boldsymbol{\rho}^{\mathrm{II}} D W_{0}^{\mathrm{II}}\right. \\
& \left.-\rho^{\mathrm{I}} D W_{0}^{\mathrm{I}}+g\left(\rho^{\mathrm{II}}-\rho^{\mathrm{I}}\right)\right)-\sigma_{u} \frac{\partial^{2} \xi_{1}}{\partial x^{2}}=0 \\
& v_{1}^{\mathrm{II}}=v_{1}^{\mathrm{III}} \\
& u_{\mathrm{i}}^{\mathrm{II}}=u_{1}^{\mathrm{III}} \\
& v_{1}^{\mathrm{II}}=\frac{\partial \eta_{1}}{\partial t} \\
& \mu^{\mathrm{III}}\left(\frac{\partial u_{1}^{\mathrm{III}}}{\partial z}+\frac{\partial v_{1}^{\mathrm{III}}}{\partial x}\right)-\mu^{\mathrm{II}}\left(\frac{\partial u_{1}^{\mathrm{II}}}{\partial z}+\frac{\partial v_{1}^{\mathrm{II}}}{\partial x}\right)=0 \\
& -p_{1}^{\mathrm{III}}+2 \mu^{\mathrm{III}} \frac{\partial v_{1}^{\mathrm{III}}}{\partial z}+p_{1}^{\mathrm{II}}-2 \mu^{\mathrm{II}} \frac{\partial v_{1}^{\mathrm{II}}}{\partial z}+\eta_{1}\left(\rho^{\mathrm{III}} D W_{0}^{\mathrm{III}}\right. \\
& \left.-\rho^{\mathrm{II}} D W_{0}^{\mathrm{II}}+g\left(\rho^{\mathrm{III}}-\rho^{\mathrm{II}}\right)\right)-\sigma_{1} \frac{\partial^{2} \eta_{1}}{\partial x^{2}}=0 .
\end{aligned}
$$


In the above equations, $\mu^{j}$ is the viscosity of phase $j$. The variable $\sigma_{\mathrm{u}}\left(\sigma_{1}\right)$ denotes the tension of an interface dividing a semiinfinite phase of I(III) from a semi-infinite phase of II. [In this analysis, variation in these surface tensions due to the surface corrugations is neglected, since this effect is small even for wavelengths of the order of film thickness (31).] The symbol " $D$ ", denotes differentiation with respect to $z$.

The set of first-order field equations and boundary conditions (Eqs. [33]- [47]) are linear and homogeneous in the unknown functions $p_{1}^{j}, u_{1}^{j}, v_{1}^{j}, \xi_{1}$, and $\eta_{1}$. (Note that the first-order potential $W_{1}^{j}$ is an integral function of $\xi_{1}$ and $\eta_{1}$, cf. Eqs. [18]- [22].) This set of equations can be solved by expanding the unknown quantities in a series of normal modes (23). The boundary conditions are composed about planes of constant $z$, and therefore a suitable form for these normal modes is the real part of the product of the function $\exp (i k x+\omega t)$ multiplied by a scalar function of $z$. Owing to the linearity and homogeneity of the first-order field and boundary equations, it is sufficient to consider only one arbitrary mode; thus

$$
\left[\begin{array}{c}
p_{1}^{j} \\
u_{1}^{j} \\
v_{1}^{j} \\
\xi_{1} \\
\eta_{1}
\end{array}\right]=\operatorname{Re}\left[\left[\begin{array}{c}
\hat{p}^{j}(z, k) \\
\hat{u}^{j}(z, k) \\
\hat{v}^{j}(z, k) \\
\hat{\xi}(k) \\
\hat{\eta}(k)
\end{array}\right] \times \exp (i k x+\omega(k) t)\right] .
$$

The parameter $k$ is the wavenumber of the normal mode (the wavelength $(\lambda)$ is equal to $2 \pi / k)$. The variable $\omega$ is the frequency of the motion (also referred to as the growth coefficient); the functional dependence of $\omega$ on $k$ is the dispersion equation. If the real part of $\omega(k)$ is greater than zero for a particular mode with wavenumber $k$, the film system is unstable to that mode since the exponential in Eq. [48] increases without bound; similarly, if $\operatorname{Re}(\omega(k))<0$, the system is stable.

The normal modes of the first order excess van der Waals potential are obtained by substituting the modal forms for $\xi_{1}$ and $\eta_{1}$ into Eqs. [21] and [22].

$$
\begin{aligned}
& \rho^{\mathrm{I}} \hat{W}_{\mathrm{I}}^{\mathrm{I}}(z)=\hat{\eta}\left(I_{\mathrm{I}, \mathrm{III}}\left(z+\frac{h}{2}, k\right)-I_{\mathrm{I}, \mathrm{II}}\left(z+\frac{h}{2}, k\right)\right) \\
& \rho^{\mathrm{II}} \hat{W}_{1}^{\mathrm{II}}(z)=\left\{\begin{array}{l}
\hat{\eta}\left(I_{\mathrm{II}, \mathrm{III}}\left(z+\frac{h}{2}, k\right)-I_{\mathrm{II}, \mathrm{II}}\left(z+\frac{h}{2}, k\right)\right) \quad\left(\frac{h}{2}>z>\frac{h}{2}-h_{1}\right) \\
\hat{\xi}\left(I_{\mathrm{II}, \mathrm{II}}\left(z-\frac{h}{2}, k\right)-I_{\mathrm{I}, \mathrm{II}}\left(z-\frac{h}{2}, k\right)\right)\left(\frac{h}{2}-h_{1}>z>-\frac{h}{2}\right)
\end{array}\right. \\
& \rho^{\mathrm{III}} \hat{W}_{1}^{\mathrm{III}}(z)=\hat{\xi}\left(I_{\mathrm{II}, \mathrm{II}}\left(z-\frac{h}{2}, k\right)-I_{\mathrm{I}, \mathrm{III}}\left(z-\frac{h}{2}, k\right)\right)
\end{aligned}
$$


where

$$
\begin{aligned}
I_{i, j}(v, k) & =\rho^{i} \rho^{j} \int_{-\infty}^{\infty} \int_{-\infty}^{\infty} \cos (k \beta) \\
& \times w_{i, j}\left[\left(v^{2}+\beta^{2}+\xi^{2}\right)^{1 / 2}\right] d \xi d \beta .
\end{aligned}
$$

Upon substituting the normal mode expressions for the dependent variables into the first-order field equations and boundary conditions, the following relations result:

$$
\begin{gathered}
\left(D^{4}-\left(2 k^{2}+\frac{\omega}{\nu^{j}}\right) D^{2}+k^{4}+\frac{k^{2} \omega}{\nu^{j}}\right) \hat{v}^{j}=0 \\
(j=\mathrm{I}, \mathrm{II} ; \mathrm{III}) \\
\lim _{z \rightarrow \infty} \hat{v}^{\mathrm{I}}=\lim _{z \rightarrow \infty} D \hat{v}^{\mathrm{I}}=0 \\
\lim _{z \rightarrow-\infty} \hat{v}^{\mathrm{III}}=\lim _{z \rightarrow-\infty} D \hat{v}^{\mathrm{III}}=0
\end{gathered}
$$

$D \hat{v}^{\mathrm{I}}=D \hat{v}^{\mathrm{II}}$

$\mu^{\mathrm{II}}\left(D^{3}-\left(3 k^{2}+\frac{\omega}{\nu^{\mathrm{II}}}\right) D\right) \hat{v}^{\mathrm{II}}-\mu^{\mathrm{I}}\left(D^{3}-\left(3 k^{2}+\frac{\omega}{\nu^{\mathrm{I}}}\right) D\right) \hat{v}^{\mathrm{I}}$

$$
-\frac{k^{4}}{\omega}\left(\left(\sigma_{u}+\frac{1}{k^{2}}\left(-\frac{d \Pi}{d h}+g\left(\rho^{\mathrm{II}}-\rho^{\mathrm{I}}\right)\right)\right) \hat{v}^{\mathrm{II}}\left(\frac{h}{2}\right)-\frac{I(h)}{k^{2}} \hat{v}^{\mathrm{II}}\left(-\frac{h}{2}\right)\right)=0
$$

$\hat{v}^{\mathrm{II}}=\hat{v}^{\mathrm{III}}$

$D \hat{v}^{\mathrm{II}}=D \hat{v}^{\mathrm{III}}$

$\left(D^{2}+k^{2}\right)\left(\mu^{\mathrm{II}} \hat{v}^{\mathrm{II}}-\mu^{\mathrm{III}} \hat{v}^{\mathrm{III}}\right)=0$

$\mu^{\mathrm{III}}\left(D^{3}-\left(3 k^{2}+\frac{\omega}{\nu^{\mathrm{III}}}\right) D\right) \hat{v}^{\mathrm{III}}-\mu^{\mathrm{II}}\left(D^{3}-\left(3 k^{2}+\frac{\omega}{\nu^{\mathrm{II}}}\right) D\right) \hat{v}^{\mathrm{II}}$
The remaining field equation (Eq. [53]) is easily integrated:

$$
\begin{aligned}
& \hat{v}^{\mathrm{II}}(z, k)=C_{1} \cosh (k z)+C_{2} \sinh (k z) \\
& \quad+C_{3} \cosh \left(k q^{\mathrm{II}} z\right)+C_{4} \sinh \left(k q^{\mathrm{II}} z\right)
\end{aligned}
$$

$\hat{v}^{\mathrm{I}}(z, k)=C_{5} \exp (-k z)$

$$
+C_{6} \exp \left(-k q^{\mathrm{I} z}\right)
$$

$$
-I_{\mathrm{I}, \mathrm{II}}(h, k)-I_{\mathrm{II}, \mathrm{III}}(h, k) .
$$

By utilizing Eqs. [31] and [52], $I(h)$ can be expressed in the following integral form:

$$
\begin{aligned}
I(h)= & h^{2} \int_{-\infty}^{\infty} \int_{-\infty}^{\infty} \cos \left(k h \beta^{\prime}\right) \\
& \times \tilde{w}\left(h\left(1+\beta^{\prime 2}+\xi^{\prime 2}\right)^{1 / 2}\right) d \xi^{\prime} d \beta^{\prime}
\end{aligned}
$$

where $\tilde{w}(r)$ is defined by Eq. [31]. where

$\hat{v}^{\mathrm{II}}(z, k)=C_{7} \exp (+k z)$

$$
+C_{8} \exp \left(+k q^{\mathrm{III}} z\right)
$$

$$
\begin{aligned}
q^{j} & =\left(1+\frac{\omega}{\nu^{j} k^{2}}\right)^{1 / 2} \\
\operatorname{Re}\left[q^{j}\right] & >0 \quad(j=\mathrm{I}, \mathrm{II}, \mathrm{III}) .
\end{aligned}
$$

The variable $\nu^{j}$ in Eq. [69] is the kinematic 
viscosity $\left(\mu^{j} / \rho^{j}\right)$ of phase $j$. Since the wavelength of perturbation, $\lambda$, characterizes a physical quantity, it can take only positive values. Therefore, in obtaining Eqs. [66][69], $k$ has been restricted to positive values and the boundary conditions at infinity (Eqs. [54] and [55]) have been used.

When the integrated field equations are substituted into the boundary conditions at $z= \pm h / 2$, a set of eight linear, homogeneous equations in the eight constants, $C_{1}-C_{8}$, are obtained. Since only nontrivial solutions to the $\hat{v}^{j}$ functions are desired, the determinant of the matrix of coefficients of the eight constants must be set equal to zero. The dispersion equation is derived from this latter condition since the elements of the matrix are functions of $k$ and $\omega$. Owing to the presence of the $\exp \left(-k q^{\mathrm{I}} z\right)$, $\exp \left(k q^{\mathrm{III}} z\right), \sinh \left(k q^{\mathrm{II}} z\right)$, and $\cosh \left(k q^{\mathrm{II}} z\right)$ terms in the integrated field equations, the dispersion equation obtained will only implicitly define $\omega$ as a function of $k$. To obtain an explicit formulation, the "viscous liquid" inequality (25) (Eq. [70]) is introduced into the dispersion equation.

$$
\frac{|\omega|}{\nu^{j} k^{2}} \ll 1 \quad(j=\text { I, II, III }) .
$$

Utilizing this inequality, the dispersion equation can be expanded in powers of $\omega / \nu^{j} k^{2}$; from this expansion, an explicit expression for $\omega$ as a function of $k$ can be obtained. Once this expression for $\omega(k)$ is derived, it can then be substituted back into Eq. [70] to verify that the inequality is satisfied. In the following section, the dispersion equation for a symmetrical system is computed. The reason for initially considering this system is that it will illustrate the following important aspect of inequality [70]: When the dispersion equation is expanded to first order in $\omega / \nu^{j} k^{2}$, the resulting expression is the same as would have been obtained had the $\partial v_{1}^{j} / \partial t$ and $\partial u_{1}^{j} / \partial t$ terms been neglected in the first-order equations of motion (Eqs. [34] and [35]). This latter observation is then used in computing the dispersion equation (accurate to first order in $\omega / \nu^{j} k^{2}$ ) of an unsymmetrical system. The validity of neglecting all terms of second order and greater in $\left(\omega / \nu^{j} k^{2}\right)$ is discussed in Appendix A.

\section{The Dispersion Equation for a Symmetrical System}

As remarked in the Introduction, a symmetrical film system is one in which gravity is neglected and in which the semi-infinite phases surrounding the film are identical. Thus, in such a system $\mu^{\mathrm{I}}=\mu^{\mathrm{III}}$, $\rho^{\mathrm{I}}=\rho^{\mathrm{II}}, q^{\mathrm{I}}=q^{\mathrm{III}}$, and $\sigma_{\mathrm{u}}=\sigma_{\mathrm{l}} \equiv \sigma$. With these simplifications, the boundary condition at $z= \pm h / 2$ admits solutions for the $\hat{v}^{j}(z, k)$ functions which are even (symmetric) and odd (antisymmetric) functions of $z$. For the even solution set $C_{7}=C_{5}$, $C_{8}=C_{6}$, and $C_{2}=C_{4}=0$; for the odd set $C_{7}=-C_{5}, C_{8}=-C_{6}$, and $C_{1}=C_{3}=0$. Substituting these solution sets into the boundary conditions at $z=h / 2$ yields a set of four linear, homogeneous equations in the four remaining unknowns. (The same four equations are generated if the sets are substituted into the boundary conditions at $z=-h / 2$.) Upon setting equal to zero the determinant of the matrix of coefficients of these four equations, the following dispersion equations for the even and odd solutions are obtained.

$$
\begin{aligned}
& \frac{\psi}{k}\left(1-\frac{\mu^{\mathrm{I}} k^{2}}{\rho^{\mathrm{II}} \omega}\left(1+q^{\mathrm{I}}\right)\left(X-q^{\mathrm{II}} Y\right)\right) \\
& +\frac{4\left(\mu^{\mathrm{II}}\right)^{2}}{\rho^{\mathrm{II}}}\left(X-q^{\mathrm{II}} Y\right)\left(1+\frac{\mu^{\mathrm{I}}}{\mu^{\mathrm{II}}}\left(q^{\mathrm{I}}-1\right)\right. \\
& \left.-\left(\frac{\mu^{\mathrm{I}}}{\mu^{\mathrm{II}}}\right)^{2} q^{\mathrm{I}}\right)+\rho^{\mathrm{II}} \frac{\omega^{2}}{k^{4}}\left(X+\frac{\rho^{\mathrm{I}}}{\rho^{\mathrm{II}}}\right) \\
& +\mu^{\mathrm{II}} \frac{\omega}{k^{2}}\left(4 X-\frac{\mu^{\mathrm{I}}}{\mu^{\mathrm{II}}} \frac{\rho^{\mathrm{I}}}{\rho^{\mathrm{II}}}\left(X-q^{\mathrm{II}} Y\right)\left(1+q^{\mathrm{I}}\right)\right) \\
& +\mu^{\mathrm{I}} \frac{\omega}{k^{2}}\left(\left(1+q^{\mathrm{I}}\right)\left(1+q^{\mathrm{II}} X Y\right)\right. \\
& \left.+2 X\left(q^{\mathrm{I}}-1\right)\right)=0
\end{aligned}
$$


where, for the even solution

$$
\begin{aligned}
& \psi=\sigma-\frac{1}{k^{2}}\left(\frac{d \Pi}{d h}+I(h)\right) \\
& X=\tanh \left(\frac{k h}{2}\right) \\
& Y=\tanh \left(\frac{k h}{2} q^{\mathrm{II}}\right)
\end{aligned}
$$

and for the odd solution

$$
\begin{aligned}
\psi & =\sigma+\frac{1}{k^{2}}\left(-\frac{d \Pi}{d h}+I(h)\right) \\
X & =\operatorname{coth}\left(\frac{k h}{2}\right) \\
Y & =\operatorname{coth}\left(\frac{k h}{2} q^{\mathrm{II}}\right) .
\end{aligned}
$$

Following the nomenclature proposed by Felderhof, the normal modes of the even solution set are termed stretching (ST) modes and those of the odd set are classified as squeezing ( $\mathrm{SQ}$ ) modes (Fig. 1). In the limit of infinite film thickness, $F(h)$ and $I(h)$ tend toward zero (cf. Eqs. [30] and [65]) and all four of the hyperbolic functions (see Eqs. [73], [74], [76], and [77]) tend to unity.
Consequently in this limit the dispersion equations for both the SQ and ST modes are identical and reduce to the dispersion equation for waves on the interface between two bulk fluids. Furthermore, the limiting expressions for the SQ and ST dispersion equations as $\mu^{1}$ and $\rho^{1}$ tend towards zero (a free liquid film) are equal to those derived by Sche (16) and Sche and Fijnaut (17). The extension to three dimensions involves only replacing $k$ by $\left(k_{x}^{2}+k_{y}^{2}\right)^{1 / 2}$, where $k_{x}$ and $k_{y}$ are the wavenumbers in the $x$ and $y$ directions, respectively (23).

Expanding the $q^{\mathrm{I}}, q^{\mathrm{II}}$, and $Y$ terms in Eq. [71] in powers of $\omega / \nu^{j} k^{2}$ and retaining only first-order terms yields the following expressions for the dispersion equation of the SQ (Eq. [78]) and ST (Eq. [79]) modes.

$$
\begin{array}{r}
\omega_{\mathrm{SQ}}=-\frac{k}{2 \mu^{\Pi}}\left(\sigma-\frac{1}{k^{2}}\left(\frac{d \Pi}{d h}-I(h)\right)\right) \\
\times \Omega_{\mathrm{SQ}}(k, h, R) \\
\omega_{\mathrm{ST}}=-\frac{k}{2 \mu^{\mathrm{II}}}\left(\sigma-\frac{1}{k^{2}}\left(\frac{d \Pi}{d h}+I(h)\right)\right) \\
\times \Omega_{\mathrm{ST}}(k, h, R)
\end{array}
$$

where

$$
\begin{aligned}
\Omega_{\mathrm{SQ}} & =\frac{\cosh (k h)+R \sinh (k h)-1-k h R}{\left(1+R^{2}\right) \sinh (k h)+2 R \cosh (k h)+k h\left(1-R^{2}\right)} \\
\Omega_{\mathrm{ST}} & =\frac{\cosh (k h)+R \sinh (k h)+1+k h R}{\left(1+R^{2}\right) \sinh (k h)+2 R \cosh (k h)-k h\left(1-R^{2}\right)} \\
R & =\frac{\mu^{\mathrm{I}}}{\mu^{\mathrm{II}}} .
\end{aligned}
$$

These results for the first-order dispersion equations are valid for both short and long wavelength disturbance; previously derived first-order equations are valid only for long wavelength perturbations $(2,4,6,16$, 17). Equations [78] and [79] could also have been derived directly by neglecting the $\omega / \nu^{j} k^{2}$ terms in the normal mode field equation (Eq. [53]) and in the normal stress boundary conditions (Eqs. [59] and [63]). This latter procedure is equivalent to neglecting the $\partial u_{1}^{j} / \partial t$ and $\partial v_{1}^{j} / \partial t$ terms in the first-order equations of motion (Eqs. [34] and [35]) and is utilized in the next section to obtain the dispersion relation for an unsymmetrical system. 
The Dispersion Equation for an Unsymmetrical System

After neglecting the $\omega / \nu^{j} k^{2}$ terms in the normal mode field equation (Eq. [53]), the following expressions for the $\hat{v}^{j}(z, k)$ functions are obtained upon integration:

$$
\begin{array}{r}
\hat{v}^{\mathrm{I}}(z, k)=a_{1} \cosh (k z)+a_{2} \sinh (k z) \\
+a_{3} z \cosh (k z)+a_{4} z \sinh (k z) \\
\hat{v}^{\mathrm{I}}(z, k)=a_{5} \exp (-k z)+a_{6} z \exp (-k z) \\
\hat{v}^{\mathrm{II}}(z, k)=a_{7} \exp (k z)+a_{8} z \exp (k z)
\end{array}
$$

where, as in Eqs. [66]-[69], $k$ has been restricted to positive values and the boundary conditions at infinity (Eqs. [54] and [55]) have been used. Upon substituting these integrated solutions into the boundary conditions at $z= \pm h / 2$ and then setting equal to zero the determinant of the resulting matrix of coefficients, the follow- ing two dispersion equations are obtained.

$$
\begin{aligned}
& \omega_{1}=\frac{k}{2 \mu^{\mathrm{II}} \delta_{2}}[\theta+\gamma] \\
& \omega_{2}=\frac{k}{2 \mu^{\mathrm{II}} \delta_{2}}[\theta-\gamma]
\end{aligned}
$$

where

$$
\begin{aligned}
\theta= & -\frac{1}{2}\left[\left(\chi_{1}-\Phi\right) \Lambda_{1}\left(R_{1}, R_{2}\right)\right. \\
& +\left(\chi_{1}+\Phi\right) \Lambda_{2}\left(R_{1}, R_{2}\right) \\
& +\left(\chi_{2}-\Phi\right) \Lambda_{1}\left(R_{2}, R_{1}\right) \\
& \left.+\left(\chi_{2}+\Phi\right) \Lambda_{2}\left(R_{2}, R_{1}\right)\right] \\
\gamma=\left\{\theta^{2}-\right. & \delta_{1} \delta_{2}\left[\left(\chi_{1}-\Phi\right)\left(\chi_{2}+\Phi\right)\right. \\
& \left.\left.+\left(\chi_{1}+\Phi\right)\left(\chi_{2}-\Phi\right)\right]\right\}^{1 / 2}
\end{aligned}
$$

The variables appearing in the above equations are defined below.

$$
\begin{aligned}
& \Lambda_{n}(\alpha, \beta)= \frac{1}{2}\left[\left(\operatorname{coth}(k h)-(-1)^{n} \operatorname{cosech}(k h)\right)\left(1-(-1)^{n} k h \operatorname{cosech}(k h)\right)\right. \\
&+\left(\beta+\alpha k^{2} h^{2} \operatorname{cosech}^{2}(k h)\right)+\beta^{2}\left(\operatorname{coth}(k h)-k h \operatorname{cosech}^{2}(k h)\right. \\
&\left.+\alpha\left(1-k^{2} h^{2} \operatorname{cosech}^{2}(k h)\right)\right)+\left(R_{1}+R_{2}\right)\left(\operatorname{coth}^{2}(k h)-(-1)^{n} \operatorname{coth}(k h)\right. \\
&\left.\times \operatorname{cosech}(k h)-(-1)^{n} k h \operatorname{cosech}(k h)\right)+R_{1} R_{2}(2 \operatorname{coth}(k h) \\
&\left.\left.-(-1)^{n} \operatorname{cosech}(k h)-(-1)^{n} k h \operatorname{coth}(k h) \operatorname{cosech}(k h)\right)\right] \quad(n=1,2) \\
& \delta_{1}=\frac{1}{2}\left[\left(R_{1}+\operatorname{coth}(k h)\right)\left(R_{2}+\operatorname{coth}(k h)\right)-\operatorname{cosech}{ }^{2}(k h)\left(1+k h R_{1}\right)\left(1+k h R_{2}\right)\right] \\
& \delta_{2}=(1-\left.k^{2} h^{2} \operatorname{cosech}(k h)\right)\left(1+R_{1}^{2} R_{2}^{2}\right)+\left(\operatorname{coth}^{2}(k h)+k^{2} h^{2} \operatorname{cosech}^{2}(k h)\right)\left(R_{1}^{2}+R_{2}^{2}\right) \\
&+2 \operatorname{coth}(k h)\left(R_{1}+R_{2}\right)+2 R_{1} R_{2}\left(1+\operatorname{coth}^{2}(k h)+\left(R_{1}+R_{2}\right) \operatorname{coth}(k h)\right)
\end{aligned}
$$

$$
\begin{gathered}
R_{1}=\frac{\mu^{\mathrm{I}}}{\mu^{\mathrm{II}}} ; \quad R_{2}=\frac{\mu^{\mathrm{III}}}{\mu^{\mathrm{II}}} \\
\chi_{1}=\sigma_{\mathrm{u}}+\frac{1}{k^{2}}\left(-\frac{d \Pi}{d h}+\left(\rho^{\mathrm{II}}-\rho^{\mathrm{I}}\right) g\right) \\
\chi_{2}=\sigma_{1}+\frac{1}{k^{2}}\left(-\frac{d \Pi}{d h}+\left(\rho^{\mathrm{III}}-\rho^{\mathrm{II}}\right) g\right) \\
\Phi=I(h) / k^{2} .
\end{gathered}
$$

These results are new in that explicit first-order dispersion equations for an unsymmetrical film system have yet to be derived; they lead to dispersion equations for a symmetrical system and for a film on a solid substrate when appropriate limits are taken. For the parameters of a symmetrical system (i.e., $\sigma_{1}=\sigma_{\mathrm{u}}, \mu^{\mathrm{I}}=\mu^{\mathrm{III}}$, $g=0$ ) Eqs. [86] and [87] reduce to Eqs. [78] and [79]. Furthermore, from the equa- 
tions for an unsymmetrical system, the dispersion relation for a wetting film (i.e., a film on a solid substrate) can be derived by computing the limiting expressions of Eqs.
[86] and [87] as $\boldsymbol{R}_{2}$ tends to infinity (Fig. 1). Depending on the sign of the $\lim _{R \rightarrow \infty} \theta$, one of the above two equations (either [86] or [87]) simplifies to $\omega^{2}=0$ as $R_{2}$ tends to infinity and the other reduces to:

where

$$
\omega=-\frac{k}{2 \mu^{\mathrm{II}}}\left(\sigma_{\mathrm{u}}+\frac{1}{k^{2}}\left(-\frac{d \Pi}{d h}+\left(\rho^{\mathrm{II}}-\rho^{\mathrm{I}}\right) g\right)\right) \Xi\left(k, h, R_{1}\right)
$$

$$
\Xi\left(k, h, R_{1}\right)=\frac{\sinh (k h) \cosh (k h)-k h+R_{1}\left(\sinh ^{2}(k h)-k^{2} h^{2}\right)}{\left(R_{1} \sinh (k h)+\cosh (k h)\right)^{2}+k^{2} h^{2}\left(1-R_{1}^{2}\right)} .
$$

Equation [97] is analogous to the result obtained by Jain and Ruckenstein (11), except for the gravitational term which was neglected in their analysis.

\section{RESULTS AND DISCUSSION}

In this section, the stability characteristics of symmetrical and unsymmetrical systems are examined, both in the long and short wavelength limits. Wherever possible, explicit asymptotic results are obtained from the general dispersion relations (Eqs. [78], [79], [86], and [87]) derived in the previous section. The results are, then, used to explain available experimental observations.

\section{Symmetrical Systems}

In this subsection, first the asymptotic forms of the dispersion equations for the squeezing and stretching modes are derived for long wavelengths (i.e., for wavenumbers satisfying the inequality $k h \ll 1$ ). Then, the complete dispersion equations (Eqs. [78] and [79]) are analyzed numerically.

(a) Asymptotic relations. A necessary prerequisite for this asymptotic development is to obtain an expression for $I(h)$ as $k \rightarrow 0$. Such an expression is obtained in Appendix $B$ and is given here in terms of II (Eq. [32]):

$I(h)=-\frac{d \Pi}{d h}-\frac{k^{2} h^{2}}{2}$

$$
\begin{gathered}
\times\left(\frac{\Pi(h)}{h}+\frac{1}{h^{2}} \int_{h}^{\infty} \Pi(\nu) d \nu\right)+O\left(k^{4}\right) \\
(k \rightarrow 0) .
\end{gathered}
$$

For a symmetrical film system Eq. [99] can be written in terms of the film tension $\Delta$, where

$$
\Delta=2 \sigma+h \Pi(h)+\int_{h}^{\infty} \Pi(\nu) d \nu
$$

Combining Eqs. [99] and [100] yields:

$$
\begin{gathered}
I(h)=-\frac{d \Pi}{d h}+k^{2}\left(\sigma-\frac{\Delta}{2}\right)+O\left((k)^{4}\right) \\
(k \rightarrow 0) .
\end{gathered}
$$

With the aid of the asymptotic development for $I(h)$, the dispersion equations can easily be simplified for the long wavelength limit.

Expanding the hyperbolic functions in the defining relations for $\Omega_{\mathrm{SQ}}$ and $\Omega_{\mathrm{ST}}$ in a Taylor series in powers of $k h$ yields the following approximate equations for $\omega_{\mathrm{SQ}}$ and $\omega_{\mathrm{ST}}$ :

$$
\begin{array}{r}
\omega_{\mathrm{SQ}} \approx-\frac{k^{3} h^{2}}{8 \mu^{\mathrm{II}}}\left(2 \sigma-\frac{\Delta}{2}-\frac{2}{k^{2}} \frac{d \Pi}{d h}\right) \\
\times\left[\frac{1+\frac{k h}{12} R}{k h+R+\frac{k^{3} h^{3}}{12} R^{2}}\right]
\end{array}
$$




$$
\left.\begin{array}{c}
\approx-\frac{k^{3} h^{2}}{8 \mu^{1}}\left(2 \sigma-\frac{\Delta}{2}-\frac{2}{k^{2}} \frac{d \Pi}{d h}\right) \\
\text { for } \frac{1}{k h} \gg R \gg k h \\
\omega_{\mathrm{ST}} \approx-\frac{k}{4 \mu^{\mathrm{II}}} \Delta \\
\times\left[\frac{1+k h R}{k^{3} h^{3}}+R+k h R^{2}\right. \\
\frac{12}{12}
\end{array}\right]
$$

In the above equations, only the first two terms in the asymptotic development of $I(h)$ are used. From Eqs. [102] and [104], the criteria for stability of the SQ and ST modes in the long wavelength limit can be formulated.

For the squeezing mode, the condition for marginal or neutral stability $(\omega=0)$ is defined by:

$$
\begin{aligned}
\sigma-\frac{1}{2} h \Pi(h)-\frac{1}{2} \int_{h}^{\infty} & \Pi(\nu) d \nu \\
& -\frac{2}{k^{2}} \frac{d \Pi}{d h}=0 .
\end{aligned}
$$

The value of $k$ which satisfies Eq. [106] is termed the critical wavenumber $\left(k_{c}\right)$. If London's law $\left(w_{i, j}(r)=-C_{i, j} r^{-6}\right)$ is utilized for the intermolecular potential, $\Pi(h)$, calculated from Eqs. [30] and [32], has the form

$$
\Pi(h)=-\frac{A}{6 \pi h^{3}} .
$$

The constant $A$ is the Hamaker constant of the film system and is defined as:

$$
A=A_{\text {I,III }}+A_{\text {II,II }}-A_{\text {I,II }}-A_{\text {II,III }}
$$

where

$$
A_{i, j}=\pi^{2} \rho^{i} \rho^{j} C_{i, j} .
$$

With $\Pi(h)$ defined by Eq. [107], the following equation for the critical wavelength $\lambda_{\mathrm{c}}\left(\lambda_{\mathrm{c}}=2 \pi / k_{\mathrm{c}}\right)$ is obtained:

$$
\lambda_{\mathrm{c}}=2 \pi\left[\frac{\pi h^{4} \sigma}{A}\right]^{1 / 2}\left[1+\frac{A}{8 \pi \sigma h^{2}}\right]^{1 / 2} .
$$

Using typical values for $\sigma\left(10^{-3}-10^{-2} \mathrm{~N} / \mathrm{m}\right)$, $A\left(10^{-20} \mathrm{~J}\right)$, and $h(10 \mathrm{~nm})$ yield values for $\lambda_{c}$ which satisfy the long wavelength restriction $\left(k_{\mathrm{c}} h \ll 1\right)$. For $\lambda>\lambda_{\mathrm{c}}, \omega_{\mathrm{SQ}}>0$ and the SQ mode is unstable; alternatively, for $\lambda<\lambda_{\mathrm{c}}, \omega_{\mathrm{SQ}}<0$ and the SQ mode is stable.

Computer simulations of $\omega_{\mathrm{SQ}}{ }^{-1}\left(=\tau_{\mathrm{SQ}}\right)$ as a function of $\lambda$ for $\lambda>\lambda_{\mathrm{c}}$ and for values of $R=1,2$, and 10 are given in Fig. 4. These simulations indicate the existence of an inflection point minimum of $\lambda_{\mathrm{SQ}}$. An analytical expression for the wavelength at which this minimum occurs is obtained by differentiating the expression for $\omega_{\mathrm{SQ}}$ (Eq. [78]) with respect to $k$ and then obtaining the roots of the equation $d \omega_{\mathrm{SQ}} / d k=0$ which satisfy the long wavelength limit. For values of $k_{\mathrm{c}}$ such that $k_{\mathrm{c}} h \ll 1$ and $1 / k_{\mathrm{c}} h>R$ $>k_{\mathrm{c}} h$, the wavelength (termed the dominant wavelength and denoted by $\lambda_{d}$ ) at which the inflection point occurs is given by:

$$
\lambda_{d} \approx 3^{1 / 2} \lambda_{c}
$$

In the limit, $R \rightarrow 0$, the dominant wavelength tends to infinity. The minimum value of $\tau_{\mathrm{SQ}}$, since it represents the largest value of $\omega_{\mathrm{SQ}}$, will dominate the growth of instabilities in the linear regime of films vibrating in the squeezing mode.

The dispersion equation given by $\mathrm{Eq}$. [105] is a new result. Equations [102], [103], [110], and [111] are, however, identical with the results of Vrij et al. (when expanded to first order in $\omega$ ) except for the term $1 / 2 \Pi(h) h+1 / 2 \int_{h}^{\infty} \Pi(\nu) d \nu$ which, for $\Pi(h)=-A / 6 \pi h^{3}$, is $-A / 8 \pi h^{2}$. This term represents the second-order term in the asymptotic development of $I(h)$ as $k \rightarrow 0$ (see Eq. [B-12]); the term is not present in the paper of Vrij et al. because their work uses the approximate disjoining pressure approach to formulate the influence of the 
van der Waals interaction on the film stability (see the Introduction).

From Eq. [104], the condition for marginal stability of the stretching mode is $\Delta$ $=0$. The system becomes unstable to the ST mode when $\Delta<0$. With $\Pi(h)$ defined by Eq. [107], the expression for the film tension is:

$$
\frac{\Delta}{2}=\sigma-\frac{A}{8 \pi h^{2}} .
$$

Thus for systems in which $\sigma<A / 8 \pi h^{2}$, the ST mode is unstable, and conversely, if $\sigma$ $>A / 8 \pi h^{2}$, the ST mode is stable. As remarked earlier, the Hamaker constant $A$ is on the order of $10^{-20} \mathrm{~J}$; using a typical value for $h$ of $10 \mathrm{~nm}$ yields a value for $A / 8 \pi h^{2}$ which is of the order of $10^{-6} \mathrm{~N} / \mathrm{m}$. Thus the stretching mode will become unstable only for systems with extremely low interfacial tensions, such as the cell membrane.

Note that the condition for marginal stability, Eq. [112], is independent of the wavelength. It should be interpreted with caution. This condition was derived using the long wavelength approximation for $I(h)$, and, therefore, cannot be used to rule out the possibility of a critical wavelength in the short wavelength limit. As a matter of fact, we will show numerically in the next subsection that a critical wavelength exists for the stretching mode in the short wavelength limit, and convince the reader that restricting the analysis to long wavelengths can, sometime, lead to misleading or incomplete results.

For the stretching mode, the dominant wavelength, in the long wavelength limit, is given by

$$
\lambda_{\mathrm{d}} \approx \frac{2 \pi h}{(6 R)^{1 / 3}}
$$

for values of $R$ much less than one $(R \ll 1)$. Thus for the ST mode, a dominant wavelength will exist for long waves only for values of $\mu^{\mathrm{I}} / \mu^{\mathrm{II}}$ which are much less than one. A significant, new conclusion evident

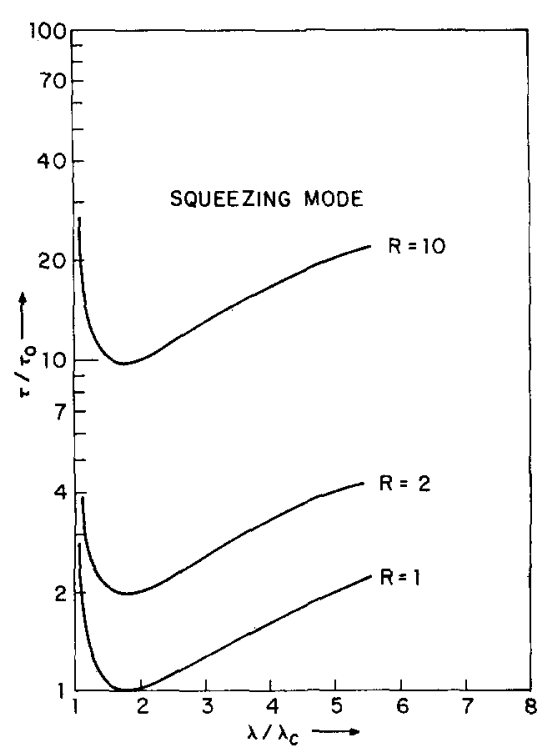

FIG. 4. Dependence of $1 / \omega(=\tau)$ on $\lambda$ for the $S Q$ mode for various values of $R$ as calculated from $\mathrm{Eq}$. [78] with Eq. [107] used for $\Pi(h)$. Note that $\tau$ is normalized with respect to $\tau_{0} . \tau_{0}$ is equal to $\tau$ evaluated at $\lambda=\lambda_{\mathrm{d}}$ and $R=1$ for the SQ mode. [ $h=50 \mathrm{mn}$, $\sigma=0.05 \mathrm{~N} / \mathrm{m}, A=10^{-20} \mathrm{~J}, \mu^{\mathrm{1}}=10^{-3} \mathrm{~kg} / \mathrm{m}$-sec.]

from Eq. [113] is that the dominant wavelength of the ST mode is a function of the hydrodynamic parameter $R$ (the ratio of viscosities) and the film thickness $h$. For the SQ mode (cf. Eq. [111]), however, $\lambda_{d}$ is independent of $R$ and is a function of $h, \sigma$, and the intermolecular interaction parameter $A$.

Using parameter characteristics of a biological membrane $\left(R=10^{-6}\right.$ and $h$ $=10 \mathrm{~nm}$ ), one finds that $\lambda_{\mathrm{d}}$ is in the order of $1 \mu \mathrm{m}$, which is the characteristic diameter of microvilli (13). The present analysis also suggests that the rate of growth of perturbations, $\omega_{\mathrm{ST}}$, is inversely proportional to membrane viscosity (Eq. [104]). Consequently, cells with a low value of membrane viscosity will form villi at a rate faster than cells with large viscosity. As a matter of fact, several investigators have suggested that the membrane of a neoplastic cell is more "fluid" (less viscous) than that of a normal cell. This increased growth rate, due to a low viscosity, could account, at least 


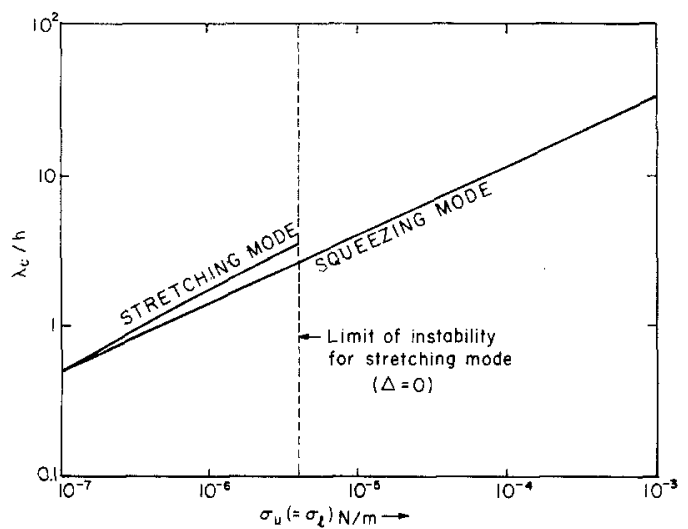

FIG. 5. Dependence of $\lambda_{c} / h$ on the surface tension for squeezing and stretching modes of vibration. Note that the stretching mode is stable for $\sigma>A / 8 \pi h^{2}$ $=4 \times 10^{-6} \mathrm{~N} / \mathrm{m}$, and that $\lambda_{\mathrm{c}, \mathrm{SQ}}<\lambda_{\mathrm{c}, \mathrm{ST}} \cdot[h=10 \mathrm{~nm}$, $A=10^{-20} \mathrm{~J}, \mu^{\mathrm{Il}}=10^{3} \mathrm{~kg} / \mathrm{m}-\mathrm{sec}, \mu^{\mathrm{I}}=\mu^{\mathrm{II}}=10^{-3} \mathrm{~kg} /$ m-sec.]

in part, for the greatly increased formation of villi in malignant cells compared to those in normal cells (13).

The stretching mode instability of a membrane can also explain ingestion and phagocytosis phenomena, in which a biological membrane bends to engulf the extracellular matter. Deformation of a biological membrane is also a key step in cellular movement. Since time constants of these processes are not available, it is not possible to compare quantitatively the results of our analysis with experimental observations, however, qualitatively our analysis offers plausible explanations of these complex biological phenomena.

(b) Numerical simulations. The cell membrane provides an example of a system in which both the squeezing and stretching modes can become unstable. Therefore, we will use parameters characteristic of the cell membrane in these numerical simulations using Eqs. [78] and [79]. The interfacial tension of the cell membrane is characteristically small (28), with typical values ranging between $10^{-6}$ and $10^{-3} \mathrm{~N} / \mathrm{m}$. Furthermore, extending into the intra- and extracellular fluids of the membrane are electrical double layers: These double layers have a destabilizing effect and can be at least qualitatively accounted for in this analysis by adding to the interfacial tension $(\sigma)$ the double layer tension $\left(\sigma_{\mathrm{d} 1}\right)$ (29). The double layer tension is of the order of $-10^{-3} \mathrm{~N} / \mathrm{m}$ for biological systems (13), and it can reduce the value of both $\sigma_{\mathrm{u}}$ and $\sigma_{1}$ considerably. Therefore, first, we will examine the role of interfacial tension $(\sigma$ $=\sigma_{\mathrm{u}}=\sigma_{1}$ ) on the stability characteristics of a membrane. Specifically, we will examine the effect of $\sigma$ on $\lambda_{c}$ for both squeezing and stretching modes.

Owing to the positive definite nature of $\Omega_{\mathrm{SQ}}$ and $\Omega_{\mathrm{ST}}$ (Eqs. [78] and [79]), critical wavenumbers $k_{\mathrm{c}}\left(=2 \pi / \lambda_{\mathrm{c}}\right)$ must satisfy the following equations:

$$
\begin{aligned}
\sigma-\frac{A}{2 \pi k_{\mathrm{c}, \mathrm{SQ} Q}^{2} h^{4}} & \\
& -\frac{A}{4 \pi h} K_{2}\left(k_{\mathrm{c}, \mathrm{SQ} Q} h\right)=0
\end{aligned}
$$

and

$$
\begin{aligned}
& \sigma-\frac{A}{2 \pi k_{\mathrm{c}, \mathrm{ST}}^{2} h^{4}} \\
& \quad+\frac{A}{4 \pi h^{2}} K_{2}\left(k_{\mathrm{c}, \mathrm{ST}} h\right)=0
\end{aligned}
$$

where $K_{2}$ is the modified Bessel function of second kind. In deriving Eqs. [114] and [115] from Eqs. [78] and [79], we have used London's law $\left(w_{i, j}=-C_{i, j} / r^{6}\right)$ to obtain expressions for $\Pi(h)\left(=-A / 6 \pi h^{3}\right)$ and $I(h)\left(=-\left(A / 4 \pi h^{2}\right) k^{2} K_{2}(k h)\right)$. Since $K_{2}(k h)$ is a monotonically decreasing, positive definite function of $k h$, a value of $k_{\mathrm{c}}$ will always exist for the squeezing mode for all values of $\sigma$. However, $k_{\mathrm{c}}$ will exist for the stretching mode only when $\sigma<A / 8 \pi h^{2}$ (or, $\Delta$ $<0$ ). The film will become stable to the stretching mode for $\Delta>0$ (Eq. [112]). In addition, if there is a critical wavenumber for the stretching mode, then one can show that for a given value of $\sigma$

$$
k_{\mathrm{c}, \mathrm{SQ}}>k_{\mathrm{e}, \mathrm{ST}} \text { or } \lambda_{\mathrm{c}, \mathrm{SQ}}<\lambda_{\mathrm{c}, \mathrm{ST}} \text {. }
$$

This condition can be obtained by subtract- 
ing Eq. [114] from Eq. [115], and realizing that $K_{2}(k h)$ is positive-definite.

The dependence of $\lambda_{\mathrm{c}} / h$ on $\sigma$ for squeezing and stretching modes is plotted in Fig. 5. Note that $\lambda_{\mathrm{c} \text {, ST }}$ ceases to exist after $\sigma=4$ $\times 10^{-6} \mathrm{~N} / \mathrm{m}$ when $\Delta>0$. When both the squeezing and stretching modes are unstable, $\lambda_{\mathbf{c}, \mathrm{ST}}>\lambda_{\mathbf{c}, \mathrm{SQ}}$, though the difference in $\lambda_{c, S T}$ and $\lambda_{c, S Q}$ is small. It must be pointed out here that $\lambda_{c, \mathrm{sQ}} / h$ agrees exactly with the value calculated using Eq. [110] for $\lambda_{\mathrm{c}} / h>10$. As mentioned in the previous section, it is not possible to calculate $\lambda_{c, s T}$ using the asymptotic expression (Eq. [112]).

Now, it is worthwhile to examine the stability characteristics of a film to perturbations of wide range of wavelengths.

In Fig. 6 is plotted computer simulations of $\omega_{\mathrm{SQ}}{ }^{-1}\left(=\tau_{\mathrm{SQ}}\right)$ and $\omega_{\mathrm{ST}}{ }^{-1}\left(=\tau_{\mathrm{ST}}\right)$ as a function of $\lambda / h$ for $1 \leq \lambda / h \leq 3000$. The maximum wavelength considered $(30 \mu \mathrm{m})$ is a typical value for the circumference of a cell membrane. A value of $10^{-6} \mathrm{~N} / \mathrm{m}$ was used for the total tension $\left(\sigma+\sigma_{\mathrm{dl}}\right)$. As expected, the numerical simulations shown in Fig. 6 are in exact agreement with the asymptotic results obtained for $\lambda / h \gg 1$ (Eqs. [102] and [104]). They, however, also lead to some interesting and new results in the limit $\lambda / h$ $\sim 1$. Most importantly, we observe a critical wavelength for the stretching mode, which was not obvious from the long wavelength analysis (Eq. [112]). Specifically for these parameter values, $\lambda_{\mathrm{c}} / h \cong 1.5$ for the squeezing mode, and $\lambda_{\mathrm{c}} / h \cong 1.75$ for the stretching mode. The minimum value of $\tau_{\mathrm{ST}}$ occurs at $\lambda_{\mathrm{d}} / h \cong 345$, exactly as predicted from the long wavelength analysis (Eq. [113]). While it is not obvious from Fig. 6 that $\tau_{\mathrm{SQ}}$ exhibits a minimum similar to those shown in Fig. 4 for $R=1,2$, and 10, its existence can be proved numerically by plotting $\tau$ versus $\lambda / h$ for values of $\lambda / h>10^{4}$. As a matter of fact, $d^{2} \tau_{\mathrm{SQ}} / d \lambda^{2}$ near $\lambda_{\mathrm{d}}$ increases as $R$ increases. This shallow minimum in the $\tau_{\mathrm{SQ}}$ versus $\lambda / h$ curve is expected since for a biological membrane, $R\left(=10^{-6}\right)$ is much less than one.

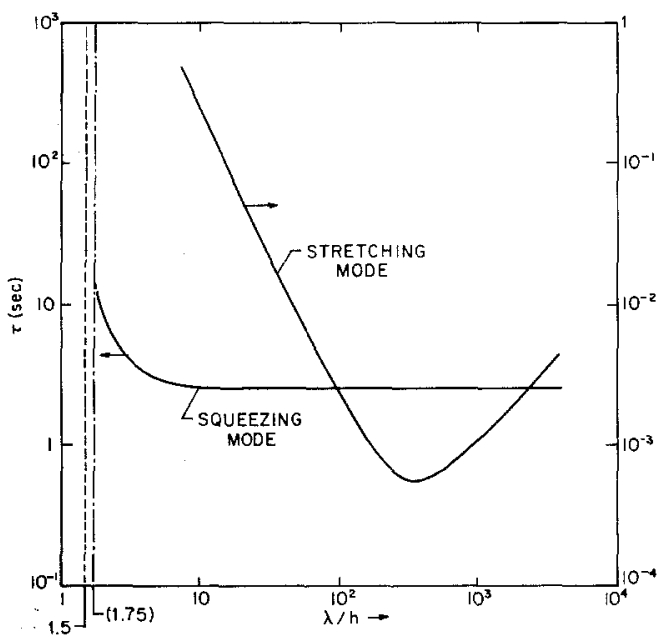

FIG. 6. Dependence of $1 / \omega(=\tau)$ on $\lambda$ for SQ and ST modes using biological parameters as calculated from Eqs. [78] and [79] with Eq. [107] used for $\Pi(h)$. [ $h$ $=10 \mathrm{~nm}, \sigma=10^{-6} \mathrm{~N} / \mathrm{m}, A=10^{-20} \mathrm{~J}, \mu^{\mathrm{II}}=10^{3} \mathrm{~kg} /$ m-sec, $\mu^{\mathrm{I}}=10^{-3} \mathrm{~kg} / \mathrm{m}$-sec.] Note that the minimum value of $\tau_{\mathrm{ST}}$ is four orders of magnitude less than the minimum value of $\tau_{\mathrm{SQ}}$.

Finally, the results indicate that both the SQ and ST modes are unstable for these parameter values and that the growth of the instabilities is dominated by the stretching mode since the minimum value of $\tau_{\mathrm{ST}}$ is four orders of magnitude less than the smallest value of $\tau_{\mathrm{SQ}}$. Physically, these simulations imply that a biological membrane will bend more readily to form microvilli or to ingest external material via phagocytosis than have "sausage type", local variation in its thickness as a result of instability (Fig. 1).

\section{Unsymmetrical Systems}

The study of the stability characteristics of unsymmetrical films is difficult because of the complexity of the general dispersion. equations. A convenient starting point for such a study is the examination of the modal forms of the first-order interfacial displacement functions $\xi_{1}$ and $\eta_{1}$. The modal forms of $\xi_{1}$ and $\eta_{1}$ are characterized by the amplitude ratio $\kappa$ and the phase difference $\phi$ between the sinusoidal vibrations of the 


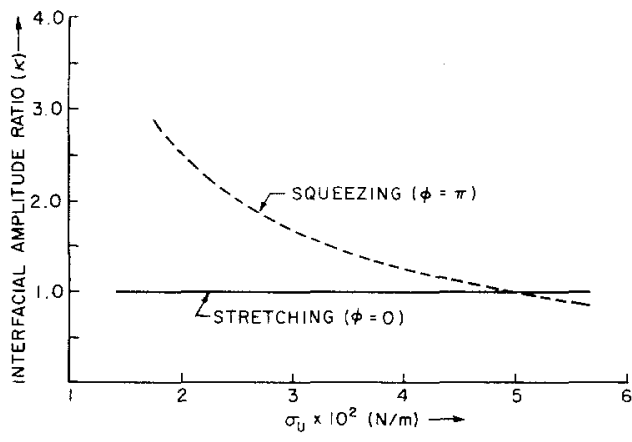

FIG. 7. Dependence of $\kappa$ on $\sigma_{u}$ in a large surface tension system for the two modes of vibration described by Eqs. [86] and [87] with Eq. [107] used for $\Pi(h) .\left[h=10 \mathrm{~nm}, \sigma_{\mathrm{l}}=0.05 \mathrm{~N} / \mathrm{m}, A=10^{-20} \mathrm{~J}\right.$, $\mu^{\mathrm{I}}=\mu^{\mathrm{II}}=\mu^{\mathrm{III}}=10^{-1} \mathrm{~kg} / \mathrm{m}-\mathrm{sec} ; \lambda=10^{2} \mu \mathrm{m}$.]

interfaces of the film. From the modal expressions for $\xi_{1}$ and $\eta_{1}$ (Eq. [48]), it is evident that

$\kappa=\frac{|\hat{\xi}|}{|\hat{\eta}|} ; \phi=\arctan \left(\frac{\operatorname{Im}[\hat{\xi} / \hat{\eta}]}{\operatorname{Re}[\hat{\xi} / \hat{\eta}]}\right)$.

The ratio $\hat{\xi} / \hat{\eta}$ is equal to $\hat{v}^{\mathrm{I}}(h / 2) / \hat{v}^{\mathrm{II}}(-h / 2)$ (cf. Eqs. [40] and [45]). An analytical expression for $\hat{v}^{\mathrm{I}}(h / 2) / \hat{v}^{\mathrm{III}}(-h / 2)$ can be obtained from the integrated field equations. To simplify the calculations, all terms containing $\omega / \nu^{j} k^{2}$ are neglected in the normal mode field equations and boundary conditions (Eqs. [53]-[63]). With this approximation, the ratio $\hat{v}^{\mathrm{I}}(h / 2) / \hat{v}^{\mathrm{II}}(-h / 2)$ is, from Eqs. [84] and [85], equal to:

$$
\begin{aligned}
& \frac{\hat{v}^{\mathrm{I}}\left(\frac{h}{2}\right)}{\hat{v}^{\mathrm{III}}\left(-\frac{h}{2}\right)} \\
& =\frac{1}{\frac{a_{7}}{a_{5}}-\frac{h}{2} \frac{a_{8}}{a_{5}}}+\frac{\frac{h}{2}}{\frac{a_{7}}{a_{6}}-\frac{h}{2} \frac{a_{8}}{a_{6}}} .
\end{aligned}
$$

From Eq. [118], it is clear that to determine the variables $\kappa$ and $\phi$, expressions for certain quotients of the integration constants are required. These expressions are obtained through the matrix of coef- ficients of the boundary conditions; details of the calculations are provided elsewhere (15). Numerical computations indicate that the variables $\kappa$ and $\phi$ depend on the interfacial tensions $\sigma_{1}$ and $\sigma_{\mathrm{u}}$ and the intermolecular forces. When the interface tension $\left(\sigma_{1}\right.$ or $\left.\sigma_{\mathrm{u}}\right)$ is large, the result is a decrease in the amplitude of the disturbance. When intermolecular forces dominate the film dynamics, the result is an increase in the amplitude of perturbation. In Figs. 7 and 8 are illustrated the variation in $\kappa$ with changes in $\sigma_{\mathrm{u}}\left(\sigma_{1}\right.$ was held constant in the simulation) for the two modes of vibration described by Eqs. [86] and [87]. For $\sigma_{1}$ $=0.05 \mathrm{~N} / \mathrm{m}, \lambda=100 \mu \mathrm{m}$, and $h=10 \mathrm{~nm}$, $\phi$ for one mode is always equal to $\pi$ (a squeezing vibration) and $\phi$ for the other mode is always equal to 0 (a stretching vibration) (Fig. 7). For the mode corresponding to a squeezing vibration ( $\phi$ $=\pi), \kappa$ decreases as $\sigma_{\mathrm{u}}$ increases. This is an expected result since the interfacial tension is inversely proportional to the deformability of an interface. For the mode corresponding to a stretching vibration $(\phi$ $=0$ ), $\kappa$ is independent of $\sigma_{\mathrm{u}}$ and remains equal to one: For $\sigma_{\mathrm{I}}=10^{-6} \mathrm{~N} / \mathrm{m}, \lambda=50$ $\mathrm{nm}$, and $h=10 \mathrm{~nm}, \phi$ for one mode is always equal to 0 (a stretching vibration) and $\phi$ for the other mode is equal to $\pi$ (a

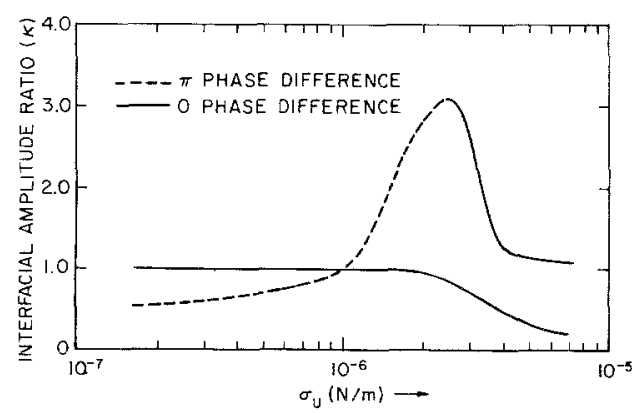

Fig. 8. Dependence of $\kappa$ on $\sigma_{u}$ in a low surface tension system for the two modes of vibration described by Eqs. [86] and [87] with Eq. [107] used for $\Pi(h) .\left[h=10 \mathrm{~nm}, \sigma_{1}=10^{-6} \mathrm{~N} / \mathrm{m}, A=10^{-20} \mathrm{~J}\right.$, $\mu^{\mathrm{I}}=\mu^{\mathrm{III}}=10^{-3} \mathrm{~kg} / \mathrm{m}-\mathrm{sec}, \mu^{\mathrm{II}}=10^{3} \mathrm{~kg} / \mathrm{m}-\mathrm{sec}, \lambda=50$ nm.] Note that for $\sigma_{\mathrm{u}}>2.5 \times 10^{-6} \mathrm{~N} / \mathrm{m}$, both modes of instability are stretching vibrations $(\phi=0)$. 
squeezing vibration) up to a certain value of $\sigma_{u}\left(=2.5 \times 10^{-6} \mathrm{~N} / \mathrm{m}\right)$ and beyond that value of surface tension, $\phi$ becomes zero again (Fig. 8). Thus, an unsymmetric membrane can have $\phi=0$ for both modes of vibration depending upon the values of surface tensions and wavelengths (Fig. 8). In this case, $\kappa$ decreases as $\sigma_{\mathrm{u}}$ increases at large values of $\sigma_{u}$ as expected. At low values of $\sigma_{\mathrm{u}}$, intermolecular forces lead to enhanced attraction at the crests of perturbations causing the amplitude ratio to increase (Fig. 8). Numerical simulations carried out for disturbances of various wavelengths showed similar trends. It must be pointed out here that in all these simulations the phase angle, $\phi$, was either 0 or $\pi$; no other values of $\phi$ were obtained since the growth coefficient, $\omega$, was always real.

Since the growth of disturbances, following the onset of instability, is governed by the dominant wavelength, $\lambda_{d}$, the latter was evaluated numerically as a function of $\sigma_{u}$ for a fixed value of $\sigma_{1}$ (Fig. 9). In these simulations parameters characteristic of a biological membrane were used to study the role of interfacial tension asymmetry on the membrane deformation. Since the dispersion equations for the unsymmetric system are complex, specific analytical generalizations are not possible. However, simulations s lown in Fig. 9 can easily be interpreted if one recalls that the value of $A / 8 \pi h^{2}$ is $4 \times 10^{-6} \mathrm{~N} / \mathrm{m}$. When either $\sigma_{1}$ or $\sigma_{\mathrm{u}}$ is less than $A / 8 \pi h^{2}$, one would expect the stretching mode to be the dominant one on the basis of our analysis of a symmetric system (Fig. 6). This is precisely what we observe in Fig. 9. When $\sigma_{1}=10^{-6}$ $\mathrm{N} / \mathrm{m}, A / 8 \pi h^{2}$ is always greater than $\sigma_{1}$, and the dominant mode is always the stretching vibration. When $\sigma_{1}=10^{-5} \mathrm{~N} / \mathrm{m}$, the dominant mode is the stretching vibration until $\sigma_{\mathrm{u}}=4 \times 10^{-6} \mathrm{~N} / \mathrm{m}$, and thereafter the dominant mode is the squeezing vibration. In addition, when $\sigma_{1}=\sigma_{\mathrm{u}}=10^{-6} \mathrm{~N} / \mathrm{m}$, $\lambda_{\mathrm{d}} / h$ is equal to 350 , as shown in Fig. 6 . When $\sigma_{\mathrm{u}}=\sigma_{1}=10^{-5} \mathrm{~N} / \mathrm{m}, \sigma$ is greater

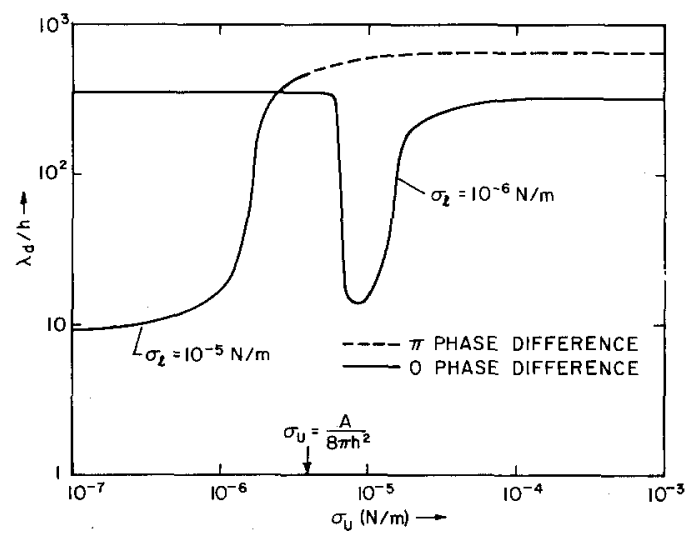

Fig. 9. Dependence of $\lambda_{\mathrm{d}} / h$ on $\sigma_{\mathrm{u}}$ for fixed values of $\sigma_{1}$ for the two modes of vibration described by Eqs. [86] and [87] with Eq. [107] used for $\Pi(h)$. [ $h$ $=10 \mathrm{~nm}, A=10^{-20} \mathrm{~J}, \mu^{\mathrm{I}}=\mu^{\mathrm{III}}=10^{-3} \mathrm{~kg} / \mathrm{m}$-sec, $\mu^{\mathrm{II}}=10^{3} \mathrm{~kg} / \mathrm{m}-\mathrm{sec}$.] Note that for $\sigma_{1}=10^{-5} \mathrm{~N} / \mathrm{m}$, the dominant mode is the stretching vibration for $\sigma_{u}$ $<4 \times 10^{-6} \mathrm{~N} / \mathrm{m}$, and the squeezing vibration for $\sigma_{\mathrm{u}}>4 \times 10^{-6} \mathrm{~N} / \mathrm{m}$.

than $A / 8 \pi h^{2}$, and the stretching mode is stable. In this case, the dominant mode is squeezing mode, and $\lambda_{\mathrm{d}} / h$ is equal to 650 .

Figure 10 shows the amplitude ratio, $\kappa_{d}$, at the dominant wavelength as a function of $\sigma_{\mathrm{u}}$ for the same fixed values of $\sigma_{\mathrm{l}}\left(10^{-6}\right.$ and $10^{-5} \mathrm{~N} / \mathrm{m}$ ) as in Fig. 9. Consider first the case when $\sigma_{1}=10^{-6} \mathrm{~N} / \mathrm{m}$ and the stretching mode is dominant for all values of $\sigma_{\mathrm{u}}$. The graph shows a critical behavior at $\sigma_{\mathrm{u}}=7$ $\times 10^{-6} \mathrm{~N} / \mathrm{m}$ : for $\sigma_{\mathrm{u}}<7 \times 10^{-6} \mathrm{~N} / \mathrm{m} \kappa_{\mathrm{d}}$ $=1$, while for $\sigma_{\mathrm{u}}>7 \times 10^{-6} \mathrm{~N} / \mathrm{m} \quad \kappa_{\mathrm{d}}$ decreases monotonically with increasing $\sigma_{u}$, a logical result. Note also that this same critical value of $\sigma_{\mathrm{u}}$ signals the rapid decline in $\lambda_{d}$ (see Fig. 9). A possible explanation for this change in the system characteristics is the fact that the critical value of $\sigma_{u}\left(7 \times 10^{-6} \mathrm{~N} / \mathrm{m}\right)$ is just greater than $A / 8 \pi h^{2}\left(4 \times 10^{-6} \mathrm{~N} / \mathrm{m}\right)$. This same phenomenon also occurs in the $\sigma_{1}=10^{-5} \mathrm{~N} / \mathrm{m}$ simulation, but right at $\sigma_{\mathrm{u}}=A / 8 \pi h^{2}=4$ $\times 10^{-6} \mathrm{~N} / \mathrm{m}$. After this value of $\sigma_{\mathrm{u}}$, both $\sigma_{\mathrm{u}}$ and $\sigma_{1}$ are greater than $A / 8 \pi h^{2}$, and consequently, as explained previously, the squeezing mode begins to dominate.

While experimental data for unsym- 


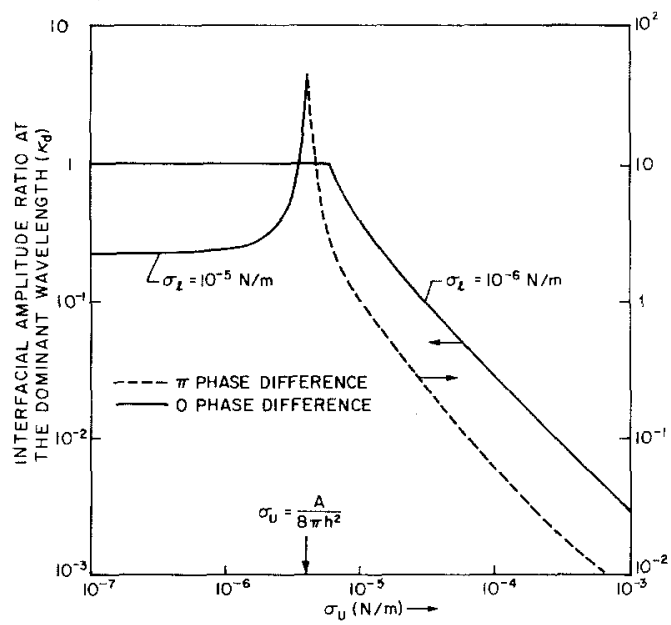

FIG. 10. Dependence of $\kappa_{d}$ on $\sigma_{u}$ for fixed values of $\sigma_{1}$. [Parameter values are the same as in Fig. 9.]

metrical systems are not available, the results we have obtained can, perhaps, suggest a mechanism which a cell uses to transmit information across its membrane. For instance, let us assume that a chemical reaction in the extra- or intracellular fluid causes the surface tension of one of the interfaces of the membrane to decrease to a value that the membrane becomes unstable. Following our analysis, we would expect that due to asymmetry in the surface tension, the amplitude ratio of disturbances $\left(\kappa_{\mathrm{d}}\right)$ may not be equal to one. The extent of deviation from one will depend upon the asymmetry in the system, and will characterize the event which has occurred in the intra- or extracellular fluid. Thus, unequal deformation of the membrane may be one possible mechanism by which a cell transmits or receives information from its surroundings.

\section{CONCLUSIONS}

The stability of thin liquid films has been studied to small perturbations using the body force approach. The analysis is more general than previous ones because it accounts for the difference in the interfacial tensions of two faces of the film and difference in the viscosities of the bounding media; it is complete because it is valid for both short and long wavelengths; and, it is systematic because it accounts for the effect of corrugations of the interface on the body force.

When the bounding phases are identical and gravity is neglected (symmetrical case), the dispersion equations suggest two modes of instability: squeezing and stretching vibrations (Fig. 1). In this case, analytical expressions are derived for the critical and dominant wavelengths, and numerical simulations are obtained for a wide range of wavelengths. The results indicate that for parameters characteristic of a biological membrane, the growth of the perturbations is dominated by the stretching mode. Physically, these simulations suggest that a biological membrane will bend more readily to form microvilli or to engulf external material, than have local variation in its thickness as seen in a squeezing vibration.

When the interfacial tensions of the two faces of the membrane are not identical, the amplitude ratio of disturbances may not be equal to one. We suggest that this deviation of the amplitude ratio from one is a possible mechanism which a cell employs to amplify or dampen a message across its membrane.

Finally, contrary to previous assertions $(16,17)$, for most cases of practical interest, the acceleration terms in the equations of motion can be neglected in a linear stability analysis of thin liquid films (Appendix A).

\section{APPENDIX A}

The objective of this appendix is to discuss the admissibility of neglecting all terms of second order and greater in $\omega /$ $\nu^{j} k^{2}$ to obtain the first-order dispersion relations. The validity of this procedure with respect to the SQ and ST dispersion equations will be discussed first. For the wavelength ranges used in the simulations exhibited in Figs. 4 through 10, the parameter $\omega / \nu^{j} k^{2}(j=\mathrm{I}$, II, III) was computed and found to be much less than one. How- 
ever, as is evident from the approximate dispersion equations of the SQ and ST modes (Eqs. [102]-[105]), the $\lim _{k \rightarrow 0}$ $\omega(k) / k^{2}$ does not converge. Consequently for extremely long waves, the approximation $\omega / \nu^{j} k^{2}$ would not be valid. Nevertheless, the values of $k$ at which $\omega(k) / k^{2}$ begins to diverge usually represent wavelengths much larger than the characteristic radii of a particle in a dispersion $(100 \mu \mathrm{m})$ or of the circumference of a biological cell $(10 \mu \mathrm{m})$ and are therefore not of interest.

Numerical simulations also indicate that the parameter $\omega / \nu^{j} k^{2}$ is not less than one for values of $R \ll 1$ and for values of the film viscosity less than or equal to the viscosity of water $\left(10^{-3} \mathrm{~kg} / \mathrm{m}-\mathrm{sec}\right)$. For these limiting cases (of which a free film is a most important example), at least the second-order term must be retained in the expansion.

The verification that the parameter $\omega /$ $\nu^{j} k^{2}$ is much less than one is not a sufficient condition for neglecting the second-order and greater terms of this parameter because the coefficients of these higher powers of $\omega / \nu^{2} k^{2}$ may be large (especially in the

long wavelength limit). Therefore the exact dispersion relations were expanded to third order in $\omega / \nu^{j} k^{2}$ and $k h$. The results indicate that the neglect of the second and third powers of $\omega / \nu^{j} k^{2}$ (when $\omega / \nu^{j} k^{2} \ll 1$ ) is unconditionally valid for the SQ mode; for the ST mode this procedure is valid without qualification only for $R \gg k h$, but it is only admissible otherwise when

and

$$
\frac{k h}{4 R}\left(\frac{\omega}{\nu^{\mathrm{II}} k^{2}}\right) \ll 1\left(\frac{k^{3} h^{3}}{12} \ll R \ll k h\right)
$$

$$
\frac{3}{k^{2} h^{2}}\left(\frac{\omega}{\nu^{\mathrm{II}} k^{2}}\right) \ll 1\left(R \ll \frac{k^{3} h^{3}}{12}\right) .
$$

Inequality [A-1] is satisfied when the kinematic viscosity of the fluid comprising the film is very large. In particular, for the stability of the stretching mode of the cell membrane (Fig. 6), inequality [A-1] is satisfied because of the large value $\left(10^{3} \mathrm{~kg} /\right.$ $\mathrm{m}-\mathrm{sec}$ ) of the viscosity of the membrane.

When $R$ is not much greater than $k h$ and inequality [A-1] is unsatisfied, the secondorder term $\omega / \nu^{\mathrm{II}} k^{2}$ must be retained in the long wavelength limit dispersion equation for the ST mode; with this correction Eq. [104] becomes:

$$
\omega_{\mathrm{ST}}=-\frac{k}{4 \mu^{\mathrm{II}}} \Delta \frac{1+k h R}{\frac{k^{3} h^{3}}{12}\left(1+3 \frac{\omega_{\mathrm{ST}}}{\nu^{\mathrm{II}} k^{4} h^{2}}\right)+R+k h R^{2}} .
$$

In general, these conclusions can be extended to the unsymmetrical system, and therefore the first-order dispersion relations for this system (Eqs. [86] and [87]) are valid (in the long wavelength limit) when $\omega / \nu^{j} k^{2}$ $\ll 1$ and $R_{1}, R_{2} \gg k h$ and when inequality [A-1] is satisfied for $R_{1}$ and $R_{2}$ not much greater than $k h$.

\section{APPENDIX B}

The objective of this appendix is to obtain an asymptotic relation for $I(h)$ as $k \rightarrow 0$. Such a relation can be obtained by substituting for the $\cos \left(k h \beta^{\prime}\right)$ in the defining relation for $I(h)$ (Eq. [65]) the following identity:

$$
\begin{aligned}
& \cos \left(k h \beta^{\prime}\right)=1-\frac{\left(k h \beta^{\prime}\right)^{2}}{2} \\
& +\left(\cos \left(k h \beta^{\prime}\right)-1+\frac{\left(k h \beta^{\prime}\right)^{2}}{2}\right) .
\end{aligned}
$$

With the above substitution, Eq. [65] becomes:

$I(h)=h^{2} \int_{-\infty}^{\infty} \int_{-\infty}^{\infty} \tilde{w}\left(h\left(1+\beta^{\prime 2}+\xi^{\prime 2}\right)^{1 / 2}\right) d \xi^{\prime} d \beta^{\prime}-\frac{1}{2} k^{2} h^{4} \int_{-\infty}^{\infty} \int_{-\infty}^{\infty} \beta^{\prime 2}$ 


$$
\begin{aligned}
\times \tilde{w}\left(h\left(1+\beta^{\prime 2}+\xi^{\prime 2}\right)^{1 / 2}\right) d \xi^{\prime} d \beta^{\prime}+h^{2} \int_{-\infty}^{\infty} \int_{-\infty}^{\infty} & \left(\cos \left(k h \beta^{\prime}\right)-1+\frac{\left(k h \beta^{\prime}\right)^{2}}{2}\right) \\
& \times \tilde{w}\left(h\left(1+\beta^{\prime 2}+\xi^{\prime 2}\right)^{1 / 2}\right) d \xi^{\prime} d \beta^{\prime} .
\end{aligned}
$$

The first integral on the right-hand side (rhs) of Eq. [B-2] can be simplified by transforming to polar coordinates:

$$
\begin{gathered}
h^{2} \int_{-\infty}^{\infty} \int_{-\infty}^{\infty} \tilde{w}\left(h\left(1+\beta^{\prime 2}+\xi^{\prime 2}\right)^{1 / 2}\right) d \xi^{\prime} d \beta^{\prime} \\
=2 \pi h^{2} \int_{0}^{\infty} \tilde{w}\left(h\left(1+r^{2}\right)^{1 / 2}\right) r d r .
\end{gathered}
$$

Defining a new variable $\nu$ by the relation $\nu=h\left(1+r^{2}\right)^{1 / 2}$ and changing the variable of integration in the integral on the rhs of Eq. [B-3] from $r$ to $\nu$ yields:

$$
\begin{gathered}
h^{2} \int_{-\infty}^{\infty} \int_{-\infty}^{\infty} \tilde{w}\left(h\left(1+\beta^{\prime 2}+\xi^{\prime 2}\right)^{1 / 2}\right) d \xi^{\prime} d \beta^{\prime} \\
=\int_{h}^{\infty} 2 \pi \nu \tilde{w}(\nu) d \nu=-\frac{d \Pi}{d h}
\end{gathered}
$$

where the second equality in Eq. [B-4] follows from Eq. [32].

After utilizing the same two transformations to rewrite the second integral on the rhs of Eqs. [B-2], the following expression is obtained:

$$
\begin{aligned}
& -\frac{1}{2} k^{2} h^{4} \int_{-\infty}^{\infty} \int_{-\infty}^{\infty} \beta^{\prime 2} \\
& \times \tilde{w}\left(h\left(1+\beta^{\prime 2}+\xi^{\prime 2}\right)^{1 / 2}\right) d \xi^{\prime} d \beta^{\prime}=-\frac{k^{2} h^{2}}{4} \\
& \quad \times\left[\frac{1}{h^{2}} \int_{h}^{\infty} 2 \pi \nu^{3} \tilde{w}(\nu) d \nu+\frac{d \Pi}{d h}\right] \cdot[\mathrm{B}-5]
\end{aligned}
$$

Integrating by parts twice the integral on the rhs of Eq. [B-5] yields:

$$
\begin{array}{r}
\int_{h}^{\infty} 2 \pi \nu^{3} \tilde{w}(\nu) d \nu=-h^{2} \frac{d \Pi}{d h}+2 h \Pi(h) \\
+2 \int_{h}^{\infty} \Pi(\nu) d \nu
\end{array}
$$

subject to the condition that

$$
\lim _{\alpha \rightarrow \infty} \alpha^{4} \tilde{w}(\alpha)=0 .
$$

Combining Eqs. [B-5] and [B-6] results in the following:

$$
\begin{aligned}
& -\frac{1}{2} k^{2} h^{4} \int_{-\infty}^{\infty} \int_{-\infty}^{\infty} \beta^{\prime 2} \\
& \times \tilde{w}\left(h\left(1+\beta^{\prime 2}+\xi^{\prime 2}\right)^{1 / 2}\right) d \xi^{\prime} d \beta^{\prime}=-\frac{k^{2} h^{2}}{2} \\
& \times\left(\frac{\Pi(h)}{h}+\frac{1}{h^{2}} \int_{h}^{\infty} \Pi(\nu) d \nu\right) \cdot \quad[\mathrm{B}-8]
\end{aligned}
$$

For the last integral on the rhs of Eq. [B-2] it can easily be shown by repeated use of L'Hôpital's rule that

$$
\begin{gathered}
h^{2} \int_{-\infty}^{\infty} \int_{-\infty}^{\infty}\left(\cos \left(k h \beta^{\prime}\right)-1+\frac{\left(k h \beta^{\prime}\right)^{2}}{2}\right) \\
\times \tilde{w}\left(h\left(1+\beta^{\prime 2}+\xi^{\prime 2}\right)^{1 / 2}\right) d \xi^{\prime} d \beta^{\prime} \\
=O\left(k^{4}\right)
\end{gathered}
$$

when the integral $A$ defined by

$$
\begin{aligned}
A= & \int_{-\infty}^{\infty} \int_{-\infty}^{\infty} \beta^{\prime 4} \\
& \times \tilde{w}\left(h\left(1+\beta^{\prime 2}+\xi^{\prime 2}\right)^{1 / 2}\right) d \xi^{\prime} d \beta^{\prime}
\end{aligned}
$$

is bounded. In Eq. [B-9], the upper case $O$ is the "large $O$ " order symbol (26). If

$$
\lim _{\alpha \rightarrow \infty} \alpha^{5+\delta} \bar{w}(\alpha)=0 \quad \delta>0,
$$

then the boundedness of $A$ is guaranteed from the " $\mu$ test" for convergence (27). Equation [B-11] is a stronger condition on the composite intermolecular potential $\bar{w}(r)$ than Eq. [B-8] and therefore this asymptotic development is restricted to intermolecular potentials which fall off faster than $r^{5+\delta}$, $\delta>0$. In particular, London's law $\left(w_{i, j}(r)\right.$ $\left.=-C_{i, j} / r^{6}\right)$ satisfies Eq. [B-11].

Combining Eqs. [B-3], [B-8], and [B-9] yields:

$I(h)=-\frac{d \Pi}{d h}-\frac{k^{2} h^{2}}{2}$ 
subject to Eq. [B-11].

A

$A_{i, j}$

$C_{i, j}$

D

F

$F_{i, j}$

$g$

$h$

$h_{1}, h_{2}$ $H_{i, j}^{1}, H_{i, j}^{2}$

$I_{i, j}$

$I(h)$

Im

$k$

$p$

$P$

$q^{\mathrm{I}}, q^{\mathrm{II}}, q^{\mathrm{III}}$

$Q_{i, j}^{1}, Q_{i, j}^{2}$

$R_{1}, R_{2}$

$R$

Re

$\mathrm{SQ}$

ST

$t$

$u$

$u_{i, j}(r)$

$$
\begin{aligned}
\times\left(\frac{\Pi(h)}{h}\right. & \left.+\frac{1}{h^{2}} \int_{h}^{\infty} \Pi(\nu) d \nu\right) \\
& +O\left((k)^{4}\right)(k \rightarrow 0)
\end{aligned}
$$

\section{APPENDIX C: NOMENCLATURE}

Hamaker constant of the film

system, defined by Eq. [108] $Y$

Hamaker constant defined by Eq. [109]

Interaction constant (between molecules $i$ and $j$ ) in London's law

$v$

$w_{i, j}(r)$

$\bar{w}(r)$

$W$

$x$

$X$

$z$

$\Delta$

$\epsilon$

Differentiation with respect to $z$

Function defined by Eq. [29]

Function defined by Eq. [2]

Acceleration of gravity

Film thickness

Lengths defined in Fig. 2

Functions defined by Eqs. [3] and [4], respectively

Function defined by Eq. [52]

Function defined by Eq. [64]

Imaginary part of a function

Wavenumber of disturbance

Pressure

Gravity-excluded total force density (defined by Eq. [26] for the base state)

Variables defined by Eq. [69]

Functions defined by Eqs. [21] and [22], respectively

Viscosity ratios defined by Eq. [93]

Viscosity ratio for a symmetrical system, defined by Eq. [82]

Real part of a function

Squeezing mode

Stretching mode

Time

$x$ component of the velocity vector

Intermolecular potential of two molecules $i$ and $j$ separated by a distance $r$ $z$ component of the velocity vector

$u_{i, j}(r)$ divided by the masses of molecules $i$ and $j$

Function defined by Eq. [31]

Excess van der Waals potential

Spatial coordinate (see Fig. 3)

Variable defined by Eq. [73] or [76]

Variable defined by Eq. [74] or [77]

Spatial coordinate (see Fig. 3)

Film tension defined by Eq. [100]

Dimensionless arbitrary parameter (much less than one) appearing in Eqs. [9]- [14]

Perturbation in lower interface

Interfacial amplitude ratio defined by Eq. [117]

Wavelength of disturbance ( $\lambda$ $=2 \pi / k)$

Viscosity

Kinematic viscosity $(\nu=\mu / \rho)$

Perturbation in upper interface

Disjoining pressure of a plane parallel, unbounded film (defined by Eq. [32])

Mass density

Interfacial tensions

Double layer tension

Reciprocal of frequency of motion $(\tau=1 / \omega)$

Phase difference defined by

Eq. [117]

Frequency of motion

\section{Subscripts}

Indicates base state value

Indicates first-order approximation

Indicates critical value

Indicates dominant value

Indicates a property of the lower (II-III) interface

Indicates a property of the upper (I-II) interface 
$i, j$

Scripted quantity is a characteristic of the interaction between molecules $i$ and $j$

\section{Superscripts}

I

II Indicates film volume

III Indicates lower semi-infinite phase

Preexponential function (see Eq. [48])

\section{ACKNOWLEDGMENTS}

This work was partially supported by the National Science Foundation, and is based on the M.S. Thesis of Charles Maldarelli.

\section{REFERENCES}

1. Vrij, A., Disc. Faraday Soc. 42, 23 (1966).

2. Felderhof, B. U., J. Chem. Phys. 49, 44 (1968).

3. Vrij, A., and Overbeek, J. Th. G., J. Am. Chem. Soc. 90, 3074 (1968).

4. Ivanov, I., Radoev, B., Manev, E., and Scheludko, A., Trans. Faraday Soc. 66, 1262 (1970).

5. Lucassen, J., van den Tempel, M., Vrij, A., and Hesselink, F., Proc. Kon. Ned. Akad. Wet. B73, 108 (1970).

6. Vrij, A., Hesselink, F., Lucassen, J., and van den Tempel, M., Proc. Kon. Ned. Akad. Wet. B73, 124 (1970).

7. Ivanov, I., and Dimitrov, D., Colloid Polym. Sci. 252, 982 (1974).

8. Ruckenstein, E., and Jain, R. K., Faraday Trans. II 70, 132 (1974).

9. Gumerman, R., and Homsy, G., Chem. Eng. Commun. 2, 27 (1975).

10. Patzer, J., and Homsy, G., J. Colloid Interface Sci. 51, 499 (1975).

11. Jain, R. K., and Ruckenstein, E., J. Colloid Interface Sci. 54, 108 (1976).

12. Ivanov, I., Doctoral Dissertation, Univ. of Sofia, Bulgaria, 1977.
13. Jain, R. K., Maldarelli, C., and Ruckenstein, E., A.I.Ch.E. Symp. Ser. Biorheol. 74, 120 (1978).

14. Joosten, J. G. H., Vrij, A., and Fijnaut, H. M., Proc. Int. Conf. on Phys. Chem. and Hydrodynamics, 1977, Hemisphere, Washington, D.C. (in press).

15. Maldarelli, C., M.S. Thesis, Columbia Univ., New York, 1978.

16. Sche, S., J. Electrostat. 5, 71 (1978).

17. Sche, S., and Fijnaut, H. M., Surface Sci. 76, 186 (1978).

18. (a) Jain, R. K., and Ivanov, I., Faraday Trans. II 76, 250 (1980). (b) Jain, R. K., Ivanov, I. B., Maldarelli, C., and Ruckenstein, E., in "Dynamics and Instability of Fluid Interfaces" " $T$. S. Sorensen, Ed.), pp. 140-167. Springer-Verlag, Berlin, 1979.

19. Scheludko, A., Proc. Kon. Ned. Akad. Wet. B65, 87 (1962).

20. Toshev, B. V., and Ivanov, I. B., Colloid Polym. Sci. 253, 558 (1975).

21. Ivanov, I. B., and Toshev, B. V., Colloid Polym. Sci. 253, 593 (1975).

22. Nir, S., Prog. Surface Sci. 8, 1 (1976).

23. Chandrasekhar, S., "Hydrodynamic and Hydromagnetic Stability." Clarendon Press, Oxford, 1961.

24. Aris, R., "Vectors, Tensors and the Basic Equations of Fluid Mechanics." Prentice-Hall, Englewood Cliffs, New Jersey, 1962.

25. Levich, V., "Physico-chemical Hydrodynamics." Prentice-Hall, Englewood Cliffs, New Jersey, 1967.

26. Nayfeh, A., "Perturbation Methods." WileyInterscience, New York, 1973.

27. Carslaw, H. S., "An Introduction to the Theory of Fourier's Sines and Integrals." Dover Publications, New York, 1950.

28. Dolowy, K., J. Theor. Biol. 52, 83 (1975).

29. Miller, C., Ph.D. Thesis, Univ. of Minnesota, Minneapolis, Minn., 1968.

30. Sanfeld, A., Steinchen, A., Dame Vedove, W., and Bisch, P. M., "Reactions in Models of Biomembranes in Connection with Cell Motion," paper presented at the American Chemical Society Meeting, Honolulu, April 1979.

31. Ono, S., and Kondo, S., "Molecular Theory of Surface Tension in Liquids," Handbuch Der Physik, Vol. 10 (S. Flugge-Marburg, Ed.), Springer-Verlag, Berlin, 1960. 